\title{
Health policy \\ Modelling the impact of physical activity on public health: a review and critique --Manuscript Draft--
}

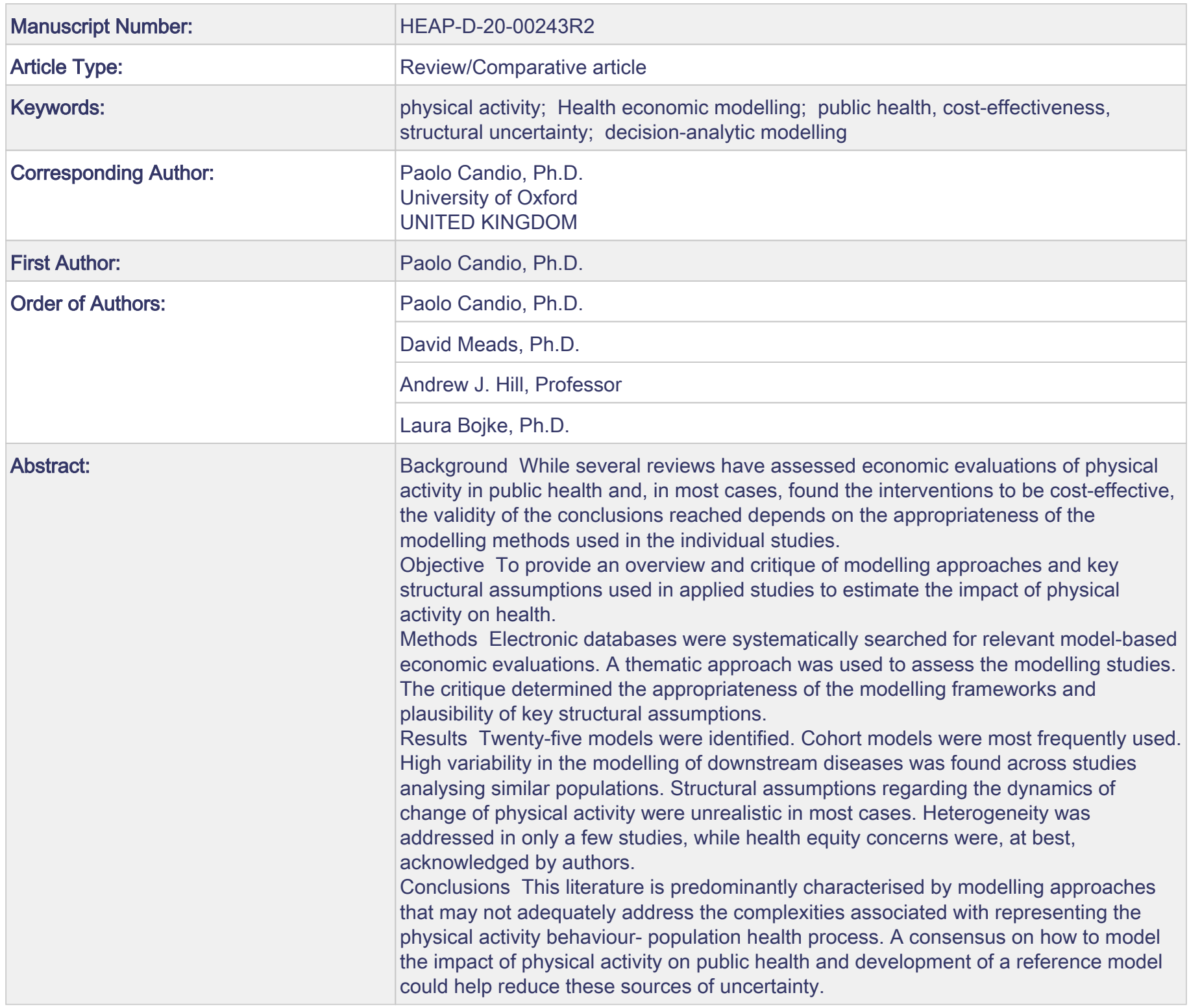


Dear Editors,

I want to thank the reviewers for their comments and suggestions. We believe that by addressing them, the article has improved substantially. We have focused the article and this is now also reflected in the title and abstract. In the Revision notes file, a detailed response to each of the reviewers' comments has been included.

Please let me know of your decision at your earliest convenience, thank you.

With my best regards,

Paolo Candio 


\section{Modelling the impact of physical activity on public health:}

\section{a review and critique}

Paolo Candio ${ }^{1,2, *}, \mathrm{PhD}$, David Meads ${ }^{2}, \mathrm{PhD}$, Andrew J. Hill ${ }^{2}, \mathrm{PhD}$, Laura Bojke ${ }^{3}, \mathrm{PhD}$,

${ }^{1}$ Health Economic Research Centre, University of Oxford, 0X37LF Oxford, UK; ${ }^{2}$ Leeds Institute of Health Sciences, University of Leeds, LS29JT Leeds UK; ${ }^{3}$ Centre for Health Economics, University of York, YO105DD Heslington UK.

Paolo Candio was supported through the White Rose PhD Studentship Network scheme as part of the National Institute for Health Research Collaboration for Leadership in Applied Health Research and Care Yorkshire and Humber (NIHR CLAHRC YH). Laura Bojke was supported by the NIHR CLAHRC YH and the NIHR Applied Research Collaboration Yorkshire and Humber (ARC YH). The views expressed in this publication are those of the authors and not necessarily those of the NHS or the Department of Health.

This manuscript has not been published elsewhere. We have no conflict of interest to disclose. Declarations of interest: none. As corresponding author, I confirm that the manuscript has been read and approved for submission by all the named authors. In terms of contributions, Paolo Candio retrieved the articles, processed the data and wrote the draft manuscript, David Meads cross-checked the included and excluded papers and all the authors contributed to the writing of the manuscript.

\footnotetext{
*Address correspondence to:

Paolo Candio, Health Economics Research Centre, University of Oxford Richard Doll Building, Old Road Campus, Oxford, OX37LF United Kingdom email: paolo.candio@ndph.ox.ac.uk.Tel.+44 7849266355
} 
Appendix 1

\section{Click here to access/download
e-component Click here to access/download
e-component
Appendix I.docx e-component
Appendix I.docx \\ Click here to access/download
e-component

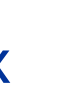

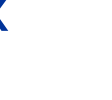

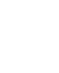 \\ Appendix l.docx}

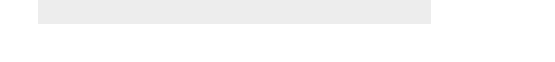

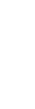

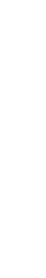




\section{Highlights}

- The common assumption of no decay of intervention effect over time is unrealistic

- Health equity concerns have not been incorporated into the models

- Development of a reference model could help reduce variability in modelling approaches 
Background While several reviews have assessed economic evaluations of physical activity in public health and, in most cases, found the interventions to be cost-effective, the validity of the conclusions reached depends on the appropriateness of the modelling methods used in the individual studies.

Objective To provide an overview and critique of modelling approaches and key structural assumptions used in applied studies to estimate the impact of physical activity on health. Methods Electronic databases were systematically searched for relevant model-based economic evaluations. A thematic approach was used to assess the modelling studies. The critique determined the appropriateness of the modelling frameworks and plausibility of key structural assumptions.

Results Twenty-five models were identified. Cohort models were most frequently used. High variability in the modelling of downstream diseases was found across studies analysing similar populations. Structural assumptions regarding the dynamics of change of physical activity were unrealistic in most cases. Heterogeneity was addressed in only a few studies, while health equity concerns were, at best, acknowledged by authors.

Conclusions This literature is predominantly characterised by modelling approaches that may not adequately address the complexities associated with representing the physical activity behaviour- population health process. A consensus on how to model the impact of physical activity on public health and development of a reference model could help reduce these sources of uncertainty.

Keywords: physical activity, economic evaluation, modelling, public health. 


\section{INTRODUCTION}

The finite resources available to decision makers dictates that commissioning of interventions ought to be based not only on the grounds of effectiveness, but also on cost-effectiveness ${ }^{1}$. To support reimbursement decisions concerned with funding interventions where there are multiple options, economic evaluation is typically used ${ }^{2}$.

Several studies have reviewed the cost-effectiveness evidence for promoting physical activity (PA) in the general population and found the interventions to provide value for money in most cases $^{3-7}$. However, methodological reviews have highlighted a number of challenges related to the economic evaluation of public health interventions, including $\mathrm{PA}^{8-11}$. Covering all elements of the evaluation, from research design of the intervention to the statistical and economic analyses, these challenges have previously been grouped into four broad categories: attribution of effects, measuring and valuing outcomes, intersectoral costs and consequences and incorporating equity concerns.

A recent review examined how the four methodological challenges above have been addressed in applied studies, across two categories of PA interventions (targeted PA and sedentary behaviour $)^{11}$. Confirming previous findings ${ }^{8-10}$, this review found an overall poor quality of reporting and marked inconsistencies in the methods applied across economic evaluations, providing a series of recommendations for the design, analysis and appraisal of economic evaluations.

The existing methodological reviews did not provide a critique on the appropriateness of the modelling approaches used to estimate the impact of changes in PA on population health. More specifically, they did not comment on the appropriateness of structural features of the modelling approach and if these aligned with the fundamental nature of the behaviourpopulation health process they were designed to represent ${ }^{12,13}$. This is important as the lack 
of adequate structure and unrealistic model assumptions can hinder the validity of costeffectiveness findings ${ }^{14}$.

\section{Challenges in modelling the impact of physical activity on public health}

More specifically, in terms of measuring effectiveness, a number of challenges in modelling healthy behaviours for public health economic evaluations, including PA, have been discussed in the literature ${ }^{14,16}$. These challenges derive from key complexities of the behaviour - population health process, which require the use of form of modelling, and include: 1) the link to multiple chronic diseases; 2) a dynamic nature of behaviour and 3) heterogeneous response to the intervention.

A recent scientific report ${ }^{17}$, which forms the basis of the current UK PA guidelines ${ }^{18}$, describes the relationship between PA and the downstream risk of disease, by assessing the relevant available evidence from systematic reviews and meta-analysis against five criteria (i.e. applicability, generalisability, risk of bias or study limitations, quantity and consistency and magnitude and precision of effect). This report confirmed that there was strong evidence suggesting a link between PA and metabolic (e.g. type II diabetes), cardiovascular (e.g. coronary heart disease and stroke), genetic mutation (e.g. colorectal and breast cancer), mental (e.g. depression) and geriatric (e.g. falls) conditions. In addition, disease probabilities may not be independent from one another. For instance, the risk of type II diabetes has been associated with risk of colorectal and breast cancers, mainly due to shared risk factors, among which PA plays a major role ${ }^{19}$.

From a modelling standpoint, this complexity represents a technical problem in that a heathy individual faces a number of different competing and complementary disease risks at any given time, based on the individuals' PA level and other personal characteristics.

Furthermore, some disease risks which will be affected by changes in PA habits in the short 
term (e.g. psychological benefits ${ }^{20}$ ), while others will take some time before occurring (e.g. colorectal cancer incidence $\left.{ }^{21}\right)$.

While PA habits generally tend to be stable over adulthood ${ }^{22}$, a decline in PA is typically associated with older age ${ }^{23}$. Evidence has also shown that natural fluctuations in PA can occur due to seasonality effects or particular life phases ${ }^{24}$. Furthermore, it is also reasonable to expect that different individuals not only will react heterogeneously, in terms of change in behaviour to the same level of exposure, but also that behaviour changes will be sustained at different rates over time ${ }^{25}$. Although the magnitude of effect will depend on the type of intervention, assuming that the changes induced by the intervention over the short term will remain constant over time is likely to be unrealistic, whereas rebound trajectories are generally more likely to occur ${ }^{26}$.

Finally, it is important to take into account heterogeneity in natural history of the PA behaviour-health process and intervention effects on this process for two main reasons: to reduce the risk of inducing bias in the cost-effectiveness results and to align the modelling approach to the objectives of the decision-makers the model is aimed to inform ${ }^{2,12}$. Public health decision-makers have a prominent goal of reducing existing health disparities in the population, for instance associated with socio-economic factors ${ }^{27}$, reflecting the value that society places on lessening unfair inequalities alongside improving health ${ }^{28,29}$.

\section{Modelling frameworks available to analysts}

There has been a wealth of research dedicated to generating taxonomies of the mathematical / epidemiological paradigms available to health economic modellers ${ }^{30-32}$. This has also reflected on their properties to inform model-based economic evaluations in public health. These frameworks have been broadly categorised into cohort and individual level approaches 
and based on their ability to formally capture time-dependent effects and interactions between individuals and the environment ${ }^{32}$.

Briefly, cohort-level approaches are generally simpler than individual level frameworks. In ascending order of modelling capacity, with decision trees and comparative risks assessments (CRAs) neither time nor interactions can be explicitly considered. Markov chains, which can be implemented at both an individual and a cohort level, can instead represent time in the process explicitly as a sequence of transitions between states. More complex are discrete time events and agent-based models which, despite their ability to formally represent changes in states over time and interactions between individuals (the latter), using either discrete and continuous time frameworks, have seen limited application in public health ${ }^{32}$.

Beside the suitability of the modelling framework, the way these methods are implemented in practice (i.e. what structural assumptions are made) can affect the validity of costeffectiveness findings ${ }^{15}$. Comparably to how the National Institute of Health and Care Excellence (NICE) evaluates models included as part of manufacturers submissions ${ }^{33}$, previews attempts to critique the plausibility of key structural assumptions of the models have been made in other public health evaluation settings ${ }^{10,16}$. To date, no methodological review has focussed on investigating these issues within the PA literature. The present paper aims to fill this gap.

\section{METHODS}

Details regarding the search strategy, eligibility criteria, study screening and selection methods are described in Appendix I. In brief, model-based economic evaluations of PA interventions were identified within the published literature, from database inception to April 2019. Given the review focus, only full economic evaluations were included (i.e. cost-utility, 
cost-benefit, cost-consequences and cost-benefit analyses). A literature search of four electronic databases (MEDLINE, EMBASE, SportDiscus and EconLit) was conducted, with free-text terms, synonyms, spelling variants, abbreviations and indexing terms related to the three concepts of economic evaluation, model and physical activity being used to identify relevant articles.

Data extraction forms were developed by adapting existing templates suggested by review guides ${ }^{34,35}$. These forms were designed to extract information on what modelling frameworks were applied and what key structural assumptions of the models relating to the three complexities discussed above (i.e. the link of physical inactivity to multiple chronic diseases; a dynamic nature of PA behaviour and heterogeneous response to the intervention) were made for estimation of the intervention impact on population health. As recommended for methodological reviews ${ }^{34,35}$, methods were summarised using a thematic approach. For reference, an overview of the modelling studies is first presented. This includes details on the decision contexts and mathematical frameworks used for economic evaluation, based on a classification recently suggested ${ }^{32}$. The second part of the review critiqued the base-case structural assumptions of the models in terms of their plausibility against the existing available evidence. While these assumptions are often interrelated, an evaluation of how the related complexities have been handled in practice is presented. The following questions thus formed the basis of the extraction process, under four themes:

- $\quad$ Modelled downstream disease risk:

$\quad$ what and how diseases associated with PA have been modelled?

- Dynamic nature of PA:

- does the model allow for natural fluctuations in PA levels over time?; 
o what assumptions have been made with regard to the link between exposure and change in PA / gains in health?;

- what assumptions have been made with regard to time-dependency of intervention effects?;

- $\quad$ Reflecting heterogeneity:

o how differences in intervention impacts between individual characteristics have been reflected?

- Incorporating health equity concerns:

- how intervention impacts on existing health inequalities have been modelled?

\section{RESULTS}

\section{Included studies}

Figure 1 shows a PRISMA flow diagram of the literature search and selection process.

Twenty-five papers met the selection criteria. Table 1 provides an overview of the modelling studies. Eleven papers based their analyses on primary data from the UK, seven from the USA, four from Australia and one each from Belgium, Canada and The Netherlands. The majority of studies focused on adults ( $>=18$ years, $n=20)$, four analyses focused only school pupils, and two included a mixed population (adults and children). Interventions based on universal approaches to promotion were explored in the majority of studies (18/25).

\section{Modelling frameworks}

Eighty percent of the studies $(n=20)$ employed aggregate-level approaches, nine of which used untimed modelling methods (eight CRAs and one decision-tree). Eleven analyses were based on discrete-time frameworks, with two multiple cohort lifetable approaches ${ }^{36,37}$, and 
Markov chain modelling being used the most frequently. Of the five individual-level models,

\section{Modelling of downstream disease risk}

Table 2 summarises the key structural assumptions made in order to estimate the impact of changes in PA on public health. The majority of studies $(n=23)$ evaluated the impact of interventions on chronic diseases and conditions associated with PA, with eight of these studies not stating which diseases were considered. The number of chronic diseases ranged from one to seven, with one study modelling 32 disease combinations ${ }^{43}$.

Except for one study which focused on Osteoporosis ${ }^{38}$, all the models selected Type II diabetes and at least one cardiovascular disease (either a type of Stroke or Coronary Heart Disease). Eleven models included at least one cancer (i.e. Colon, Colorectal, Breast, Lung and Kidney) and only two studies considered impacts on mental health outcomes, specifically, depression. Only one study ${ }^{44}$ included exercise-related injuries among the consequences. For those studies focussing on adults from the general population $(n=14)$, the majority $(\mathrm{n}=8)$ selected five chronic conditions. In addition, while the models evolved over time in terms of disease selection, except for one study which applied random search method to calibrate disease risk parameters ${ }^{42}$, none of the models took into account synergistic and compensatory effects between disease risks.

Thus, none of the reviewed models would be able to reflect the currently available evidence on the associated chronic disease risk, therefore, not adequately capturing the impact of 
changes in PA, either due to a narrow evaluation scope and/or lack of adjustments for interaction between diseases.

\title{
Dynamics of physical activity
}

\author{
Natural course of PA \\ Except for two studies that developed a natural history model from PA available from a \\ country-level surveillance system ${ }^{45} 46$, none of the models accounted for natural fluctuations \\ in PA levels over time. Baseline PA states were assumed to be stable, also when long-term \\ time horizons were considered, 15/25 evaluations with time horizons equal to or longer than \\ 30 years). Furthermore, transitions between the highest and the lowest levels of PA were not \\ allowed.
}

\section{Link between exposure and response and its time dependency}

All but four models reported that changes in PA and related health gains would be immediate following exposure to the intervention. One study ${ }^{47}$ assumed that the intervention could not affect disease risk in the first year ("run-in period"). Other analyses assumed that it would take two years ${ }^{48}$, or up to five years ${ }^{49}$, for the intervention to reach full effect. Except for one report which reported on the time lag assumed between changes in PA and disease occurrence used in the model ${ }^{41}$, none of the other studies provides details regarding time lag to disease.

\section{Time-dependent intervention effects}

The majority of models (15/25) assumed implicitly or explicitly that the intervention effect would not decay after the intervention ended (i.e. beyond follow-up assessment period). The remaining 10 analyses assumed a constant and homogeneous decline in effect, ranging from $25 \%$ to $100 \%$, up to two years after the intervention ended. 
Although lack of data may have driven these choices, the structural assumptions related to the dynamics of change in PA that underlie the current models are underreported and may be unrealistic. While appropriate sensitivity analysis may have helped characterise these complexities for decision-making ${ }^{2}$, only a minority of models explored the impact of variations to these base-case structural assumptions (see Table 2).

\section{Reflecting heterogeneity}

Ten studies used population average approaches, evaluating the health impact of changes in PA levels in homogeneous groups of inactive / sedentary adults or school pupils. Baseline differences in PA were considered in only nine studies. Three to five levels (i.e. PA states) were defined in these analyses, with part of the models aligning the classification of PA levels to current national-level PA recommendations ${ }^{37,45,46,51-53}$. Twelve studies accounted for heterogeneous health impacts based on at least age or gender, with two analyses also considering ethnicity/race differences ${ }^{40,42}$.

Thus, while some degree of heterogeneity has been captured by the reviewed models, only a minority addressed this aspect formally by considering at least basic socio-demographic differences, and in particular, baseline PA level.

\section{Incorporating health equity}

None of the reviewed economic models incorporated concerns relating to the distributional impact of the intervention formally into the economic evaluation. Where models accounted for heterogeneous effects, they did not report subgroup analysis which would have allowed for inferences about effects on health inequalities. In only a minority of studies (5/25) equity considerations were discussed. 


\section{DISCUSSION AND CONCLUSIONS}

\section{Main findings}

Complementary to previous reviews ${ }^{8-11,14}$, this is the first methodological review to provide an overview and critique of the modelling approaches used in model-based economic evaluations for estimating impacts of changes in PA on public health. This review has shed light on key structural assumptions that underlie the models, which can aid interpretation of the cost-effectiveness findings and highlights model development opportunities.

If, on one hand, economic evaluation guidelines support the notion that model structures should be kept as simple as possible $e^{54,55}$, models should align to the nature of the process they are intended to represent ${ }^{12}$. While acknowledging that the trade-off between simplicity and internal validity still represents an unresolved challenge for modellers, this literature is predominantly characterised by modelling approaches that may not adequately address the complexities associated with the PA behaviour - population health process they were intended to represent. In addition, key structural assumptions were often unsupported by relevant evidence and based on general rules of thumb.

\section{Minimum modelling standards}

Based on the findings from this review, there are a number of areas in which minimum modelling standards should be established. First, modelling of downstream disease risks. The disease selection in the reviewed models does not reflect the current available epidemiological evidence on the relationship between PA and chronic disease. While differences in environmental-level factors may explain differences in magnitude of risk between settings (e.g. countries), disease selection for identified populations should be only based on strong and updated epidemiological evidence. Disease risks are not likely to be 
independent from one another, and this should be taken into account, either formally (e.g. using calibration methods) or informally (e.g. by means of scenario analysis).

Second, accommodating for the dynamics of PA. Natural trends in PA levels should be used as baseline data for comparison. Conceptualising PA levels as fixed states is likely to induce bias in the estimations, especially when modelling PA behaviours that are subject to seasonality effects (e.g. gym attendance in summer) or over sensitive life phases (e.g. retirement). However, in scenarios where PA levels are likely to be stable, the decision to incorporate this element into the model needs to be evaluated in light of the trade-off between added complexity and accuracy.

Assumptions regarding time lags and decay of intervention effects over time must also be made explicit. The current lack of data on long-term impacts of PA intervention does not justify the use of unrealistic assumptions. Instead, modelling of PA should include analysis of a range of possible scenarios for adequately reflecting the uncertainty surrounding the decision $^{2}$. The common assumption found across the models of no decay of effect over time is unrealistic, especially when assessing effects over long periods of time. This is likely to result in overestimation of the health benefits of the intervention and undermine the validity of research findings.

Third, reflecting heterogeneity. Models should capture systematic differences in impacts between individual characteristics ${ }^{56}$. Moderator variables should be identified both for the estimation of effectiveness and cost-effectiveness of PA interventions directed at heterogeneous populations. Although sub-group analyses will not always be feasible in practice (e.g. due to small sample sizes), reports should make explicit the reasons for not conducting them. Reports should also include details regarding the gradients in risks between population sub-groups, and how the shape of these gradients is assumed (e.g. curvilinear ${ }^{57}$ ) when no data were available. 
Forth, on the issue of incorporating health inequity concerns. Methods for incorporating such

\section{Limitations}

This review is subject to several limitations. The level of detail presented in the applied studies leads us to conclude that an overall poor quality of reporting was observed, making the assessment of the modelling studies difficult. However, a formal quality assessment was not conducted according to previously defined criteria, limiting the ability of this review to determine a more traditional judgement on study quality. In addition, whilst a detailed systematic search was conducted, this review only included PA interventions, therefore 
limiting the generalisability of the findings to economic evaluations of interventions where PA is combined with other technologies or healthy behaviours.

This study focused on the cost-effectiveness literature only, and it is possible that other types of studies, such as epidemiological studies, have addressed some of the complexities discussed. Furthermore, methodological aspects discussed here represent only a proportion of the challenges that are apparent when modelling of impact of different types of PA interventions. Specifically, modelling issues related to compensatory or synergistic effects with other behaviours on the pathway to health improvement, such as dietary patterns, interactions between individuals, and non-health effects of these interventions, have not been addressed in this review and should be the focus of further research.

\section{Concluding remarks}

Reaching a consensus on minimum modelling standards, improved reporting of the key model assumptions using the available reporting checklists ${ }^{61-64}$ and development of a reference model ${ }^{65}$ could help reduce these sources of uncertainty, and therefore support optimal decision-making. 


\section{REFERENCES}

1. National Institute for Health and Care Excellence. Methods for the development of NICE public health guidance (third edition) 2012 [Available from:

https://www.nice.org.uk/process/pmg4/resources/methods-for-the-development-ofnice-public-health-guidance-third-edition-pdf-2007967445701.

2. Briggs A, Sculpher, M., Claxton, K.,. Decision Modelling for Health Economic Evaluation. Oxford: Oxford University Press 2006.

3. Garrett S, Elley CR, Rose SB, et al. Are physical activity interventions in primary care and the community cost-effective? A systematic review of the evidence. Br J Gen Pract 2011;61(584):e125-33. doi: 10.3399/bjgp11X561249 [published Online First: 2011/03/08]

4. Gc V, Wilson EC, Suhrcke M, et al. Are brief interventions to increase physical activity cost-effective? A systematic review. Br J Sports Med 2016;50(7):408-17. doi: 10.1136/bjsports-2015-094655 [published Online First: 2015/10/07]

5. Gordon L, Graves N, Hawkes A, et al. A review of the cost-effectiveness of face-to-face behavioural interventions for smoking, physical activity, diet and alcohol. Chronic Illn 2007;3(2):101-29. doi: 10.1177/1742395307081732 [published Online First: 2007/12/18]

6. Laine J, Kuvaja-Kollner V, Pietila E, et al. Cost-effectiveness of population-level physical activity interventions: a systematic review. Am J Health Promot 2014;29(2):71-80. doi: 10.4278/ajhp.131210-LIT-622 [published Online First: 2014/11/02]

7. Pavey TG, Anokye N, Taylor AH, et al. The clinical effectiveness and cost-effectiveness of exercise referral schemes: a systematic review and economic evaluation. Health Technol Assess 2011;15(44):i-xii, 1-254. doi: 10.3310/hta15440 [published Online First: 2011/12/21]

8. Weatherly H, Drummond M, Claxton K, et al. Methods for assessing the cost-effectiveness of public health interventions: key challenges and recommendations. Health Policy 2009;93(2-3):85-92. doi: 10.1016/j.healthpol.2009.07.012 [published Online First: $2009 / 08 / 28]$

9. Alayli-Goebbels AF, Evers SM, Alexeeva D, et al. A review of economic evaluations of behavior change interventions: setting an agenda for research methods and practice. J 
Public Health (Oxf) 2014;36(2):336-44. doi: 10.1093/pubmed/fdt080 [published Online First: 2013/08/24]

10. Griffiths UK, Anigbogu B, Nanchahal K. Economic evaluations of adult weight management interventions: a systematic literature review focusing on methods used for determining health impacts. Appl Health Econ Health Policy 2012;10(3):145-62. doi: 10.2165/11599250-000000000-00000 [published Online First: 2012/03/24]

11. Cochrane M, Watson PM, Timpson H, et al. Systematic review of the methods used in economic evaluations of targeted physical activity and sedentary behaviour interventions. Soc Sci Med. 2019;232:156-167. doi:10.1016/j.socscimed.2019.04.040

12. Squires H, Chilcott J, Akehurst R, Burr J, Kelly MP. A Framework for Developing the Structure of Public Health Economic Models. Value Health. 2016;19(5):588-601. doi:10.1016/j.jval.2016.02.011

13. Candio, P. 2019. Economic evaluation of universal programmes to promote healthy behaviours: challenges and possible solutions with an application to physical activity (Doctoral thesis).

[online :http://etheses.whiterose.ac.uk/26439/1/PhD\%20thesis\%20document\%20$\% 20$ candio.pdf]

14. Squires H, Chilcott J, Akehurst R, Burr J, Kelly MP. A systematic literature review of the key challenges for developing the structure of public health economic models. Int $\mathrm{J}$ Public Health. 2016;61(3):289-298. doi:10.1007/s00038-015-0775-7

15. Tsoi B, Jegathisawaran J, Tarride J-E, Blackhouse G, O’Reilly D (2015) Do different decision-analytic modelling approaches produce different cost-effectiveness results? Insights from a systematic review of existing cross-validation studies. Expert Rev Pharmacoecon Outcomes Res 15(3):451-463

16. Tsoi B, O'Reilly D, Jegathisawaran J, Tarride JE, Blackhouse G, Goeree R. Systematic narrative review of decision frameworks to select the appropriate modelling approaches for health economic evaluations. BMC Res Notes. 2015;8:244. Published 2015 Jun 17. doi:10.1186/s13104-015-1202-0

17. Office of Disease Prevention and Health Promotion. Physical Activity Guidelines Advisory Committee Scientific Report 2018 [Available from: https://health.gov/paguidelines/secondedition/report/pdf/04_C_Background_and_Key_Physical_Activity_Concepts.pdf. 
18. Chief Medical Officers. UK Chief Medical Officers' Physical Activity Guidelines 2019 [Available from:

https://assets.publishing.service.gov.uk/government/uploads/system/uploads/attachme nt_data/file/832868/uk-chief-medical-officers-physical-activity-guidelines.pdf.

19. Giovannucci E, Harlan DM, Archer MC, et al. Diabetes and Cancer: A Consensus Report. Ca-Cancer J Clin 2010;60(4):207-21. doi: 10.3322/caac.20078

20. Mandolesi L, Polverino A, Montuori S, et al. Effects of Physical Exercise on Cognitive Functioning and Wellbeing: Biological and Psychological Benefits. Front Psychol 2018;9 doi: ARTN 509 10.3389/fpsyg.2018.00509

21. Oruc Z, Kaplan MA. Effect of exercise on colorectal cancer prevention and treatment. World J Gastro Oncol 2019;11(5):348-66. doi: 10.4251/wjgo.v11.i5.348

22. Cleland V, Dwyer T, Venn A Which domains of childhood physical activity predict physical activity in adulthood? A 20-year prospective tracking study British Journal of Sports Medicine 2012;46:595-602.

23. Westerterp KR. Changes in physical activity over the lifespan: impact on body composition and sarcopenic obesity. Obes Rev 2018;19 Suppl 1:8-13. doi: 10.1111/obr.12781 [published Online First: 2018/12/05]

24. Tucker P, Gilliland J. The effect of season and weather on physical activity: a systematic review. Public Health 2007;121(12):909-22. doi: 10.1016/j.puhe.2007.04.009 [published Online First: 2007/10/09]

25. Van Dyck D, Mertens L, Cardon G, et al. Opinions Toward Physical Activity, Sedentary Behavior, and Interventions to Stimulate Active Living During Early Retirement: A Qualitative Study in Recently Retired Adults. J Aging Phys Act 2017;25(2):277-86. doi: 10.1123/japa.2015-0295 [published Online First: 2016/10/21]

26. Neil Howlett, Daksha Trivedi, Nicholas A Troop, Angel Marie Chater, Are physical activity interventions for healthy inactive adults effective in promoting behavior change and maintenance, and which behavior change techniques are effective? A systematic review and meta-analysis, Translational Behavioral Medicine, Volume 9, Issue 1, February 2019, Pages 147-157, https://doi.org/10.1093/tbm/iby010

27. Marmot M. Fair Society, Healthy Lives The Marmot Review 2010 [Available from: https://www.parliament.uk/documents/fair-society-healthy-lives-full-report.pdf. 28. Exworthy, M, Oliver, M. Evidence and health inequalities: the Black, Acheson and Marmot reports. In: Exworthy, M, Peckham, S, Powell, M, Hann, A, eds. Shaping Health Policy Case Study Methods and Analysis. Bristol, UK: Policy Press; 2012. 
29. Guindo, LA, Wagner, M, Baltussen, R. From efficacy to equity: literature review of decision criteria for resource allocation and healthcare decisionmaking. Cost Effectiveness Resource Allocation. 2012;10(1):9.

30. Brennan A, Chick SE, Davies R (2006) A taxonomy of model structures for economic evaluation of health technologies. Health Econ 15:1295-1310

31. Chick SE (2007) Taxonomy of model structure for health economics. http://www2.wmin.ac.uk/hscmg/qmmhealth2007/talks/Chick_S.IMAHealth2007..Keynote.pdf. Accessed 20 Jan 2014

32. Briggs AD, Wolstenholme J, Blakely T, et al. Choosing an epidemiological model structure for the economic evaluation of non-communicable disease public health interventions. Popul Health Metr 2016;14:17. doi: 10.1186/s12963-016-0085-1 [published Online First: 2016/05/07]

33. National Institute for Health and Care Excellence. Guide to the processes of technology appraisal, 2018. [Available from: https://www.nice.org.uk/process/pmg19/chapter/the-appraisal-process\#evidencereview.

34. Centre for Reviews and Dissemination. Systematic Reviews CRD's guidance for undertaking reviews in health care: CRD, University of York; 2009 [Available from: https://www.york.ac.uk/media/crd/Systematic_Reviews.pdf.

35. Joanna Briggs Institute. Joanna Briggs Institute Reviewer's Manual Adelaide: University of Adelaide; 2017 [Available from: https://reviewersmanual.joannabriggs.org/

36. Cobiac LJ, Vos T, Barendregt JJ. Cost-Effectiveness of Interventions to Promote Physical Activity: A Modelling Study. Plos Medicine 2009;6(7) doi: ARTN e1000110 10.1371/journal.pmed.1000110

37. Zapata-Diomedi B, Knibbs LD, Ware RS, et al. A shift from motorised travel to active transport: What are the potential health gains for an Australian city? PLoS ONE 2017;12(10):e0184799. doi: https://dx.doi.org/10.1371/journal.pone.0184799

38. Nshimyumukiza L, Durand A, Gagnon M, et al. An economic evaluation: Simulation of the cost-effectiveness and cost-utility of universal prevention strategies against osteoporosis-related fractures. J Bone Miner Res 2013;28(2):383-94. doi: 10.1002/jbmr.1758 [published Online First: 2012/09/20]

39. Goyder E, Hind D, Breckon J, et al. A randomised controlled trial and cost-effectiveness evaluation of 'booster' interventions to sustain increases in physical activity in middle- 
aged adults in deprived urban neighbourhoods. Health Technology Assessment 2014;18(13):1-+. doi: 10.3310/hta18130

40. Guo JY, Gandavarapu S. An economic evaluation of health-promotive built environment changes. Prev Med 2010;50 Suppl 1:S44-9. doi: 10.1016/j.ypmed.2009.08.019 [published Online First: 2009/10/21]

41. Cradock AL, Barrett JL, Kenney EL, et al. Using cost-effectiveness analysis to prioritize policy and programmatic approaches to physical activity promotion and obesity prevention in childhood. Prev Med 2017;95:S17-S27. doi:

10.1016/j.ypmed.2016.10.017

42. Gc VS, Suhrcke M, Hardeman W, et al. Cost-Effectiveness and Value of Information Analysis of Brief Interventions to Promote Physical Activity in Primary Care. Value Health 2018;21(1):18-26. doi: 10.1016/j.jval.2017.07.005 [published Online First: 2018/01/07]

43. Gulliford M, Charlton J, Bhattarai N, et al. Social and material deprivation and the costeffectiveness of an intervention to promote physical activity: cohort study and Markov model. Journal of Public Health 2014;36(4):674-83. doi: 10.1093/pubmed/fdt132

44. Munro JF, Nicholl JP, Brazier JE, et al. Cost effectiveness of a community based exercise programme in over 65 year olds: cluster randomised trial. J Epidemiol Commun $\mathrm{H}$ 2004;58(12):1004-10. doi: 10.1136/jech.2003.014225

45. Roux L, Pratt M, Lee IM, et al. Does age modify the cost-effectiveness of communitybased physical activity interventions? J Phys Act Health 2015;12(2):224-31. doi: 10.1123/jpah.2013-0167 [published Online First: 2014/05/20]

46. Roux L, Pratt M, Tengs TO, et al. Cost effectiveness of community-based physical activity interventions. Am J Prev Med 2008;35(6):578-88. doi: 10.1016/j.amepre.2008.06.040 [published Online First: 2008/11/13]

47. Anokye NK, Lord J, Fox-Rushby J. Is brief advice in primary care a cost-effective way to promote physical activity? Brit J Sport Med 2014;48(3):202-06. doi: 10.1136/bjsports-2013-092897

48. Barrett JL, Gortmaker SL, Long MW, et al. Cost Effectiveness of an Elementary School Active Physical Education Policy. American Journal of Preventive Medicine 2015;49(1):148-59. doi: 10.1016/j.amepre.2015.02.005

49. Cavill N, Muller, L., Mulhall, C. \& Rutter, H. Cycling demonstration towns: A costeffective investment to promote physical activity. Obesity Reviews 2011;12 
50. Dallat MAT, Soerjomataram I, Hunter RF, et al. Urban greenways have the potential to increase physical activity levels cost-effectively. Eur J Public Health 2014;24(2):19095. doi: 10.1093/eurpub/ckt035

51. Frew EJ, Bhatti M, Win K, et al. Cost-effectiveness of a community-based physical activity programme for adults (Be Active) in the UK: an economic analysis within a natural experiment. Br J Sports Med 2014;48(3):207-12. doi: 10.1136/bjsports-2012091202 [published Online First: 2012/07/17]

52. Over EA, Wendel-Vos GW, van den Berg M, et al. Cost-effectiveness of counseling and pedometer use to increase physical activity in the Netherlands: a modeling study. Cost Eff Resour Alloc 2012;10(1):13. doi: 10.1186/1478-7547-10-13 [published Online First: 2012/09/26]

53. Pringle A, Cooke C, Gilson N, et al. Cost-effectiveness of interventions to improve moderate physical activity: A study in nine UK sites. Health Education Journal 2010;69(2):211-24. doi: 10.1177/0017896910366790

54. Stahl JE 2008. Modelling methods for pharmacoeconomics and health technology assessment: an overview and guide. Pharmacoeconomics 26:131-148

55. Sculpher M, Fenwick E, Claxton K 2000. Assessing quality in decision analytic cost-effectiveness models. A suggested framework and example of application. Pharmacoeconomics 17:461-477

56. Vanni T, Karnon J, Madan J, et al. Calibrating models in economic evaluation: a sevenstep approach. Pharmacoeconomics 2011;29(1):35-49. doi: 10.2165/11584600000000000-00000 [published Online First: 2010/12/15]

57. Lacey B, Golledge J, Yeap BB, et al. Physical activity and vascular disease in a prospective cohort study of older men: The Health In Men Study (HIMS). BMC geriatr 2015;15:164. doi: 10.1186/s12877-015-0157-2 [published Online First: $2015 / 12 / 15]$

58. Sculpher MJ, Claxton K, Drummond M, et al. Whither trial-based economic evaluation for health care decision making? Health Economics 2006;15(7):677-87. doi: 10.1002/hec. 1093

59. Cookson R, Drummond, M., \& Weatherly, H. Explicit incorporation of equity considerations into economic evaluation of public health interventions. Health Economics, Policy and Law 2009;4(2):231-45. doi: https://doi.org/10.1017/S1744133109004903 
60. National Institute for Health and Care Excellence. Guide to the methods of technology appraisal 20132013 [Available from:

https://www.nice.org.uk/process/pmg9/chapter/the-reference-case\#exploringuncertainty.

61. Evers S, Goossens M, de Vet H, van Tulder M, Ament A. Criteria list for assessment of methodological quality of economic evaluations: Consensus on Health Economic Criteria. Int J Technol Assess Health Care. 2005;21(2):240-245.

62. Ghislaine A.P.G. van Mastrigt, Mickaël Hiligsmann, Jacobus J.C. Arts, Pieter H. Broos, Jos Kleijnen, Silvia M.A.A. Evers \& Marian H.J.M. Majoie (2016) How to prepare asystematic review of economic evaluations for informing evidence-based healthcare decisions: a five-step approach (part 1/3), Expert Review of Pharmacoeconomics \& Outcomes Research, 16:6, 689-704, DOI: 10.1080/14737167.2016.1246960

63. Philips Z, Bojke L, Sculpher M, Claxton K, Golder S. Good practice guidelines for decision-analytic modelling in health technology assessment: a review and consolidation of quality assessment. Pharmacoeconomics. 2006;24(4):355-371. doi:10.2165/00019053-200624040-00006

64. Husereau D, Drummond M, Petrou S, et al. Consolidated health economic evaluation reporting standards (CHEERS) — explanation and elaboration: a report of the ISPOR Health Economic Evaluations Publication Guidelines Good Reporting Practices Task Force. Value Health. 2013;16(2):231-250.

65. Haji Ali Afzali H, Bojke L, Karnon J. Improving Decision-Making Processes in Health: Is It Time for (Disease-Specific) Reference Models? Appl Health Econ Health Policy 2019 doi: 10.1007/s40258-019-00510-6 [published Online First: 2019/08/23]

66. Amarasinghe AK. Cost-effectiveness implications of GP intervention to promote physical activity: evidence from Perth, Australia. Cost Eff Resour Alloc 2010;8:10. doi: 10.1186/1478-7547-8-10 [published Online First: 2010/05/14]

67. Beale SJ, Bending MW, Trueman P, et al. Should we invest in environmental interventions to encourage physical activity in England? An economic appraisal. Eur J Public Health 2012;22(6):869-73. doi: 10.1093/eurpub/ckr151

68. De Smedt D, De Cocker K, Annemans L, et al. A cost-effectiveness study of the community-based intervention '10 000 Steps Ghent'. Public Health Nutrition 2012;15(3):442-51. doi: 10.1017/S1368980011001716

69. Montes F, Sarmiento OL, Zarama R, et al. Do Health Benefits Outweigh the Costs of Mass Recreational Programs? An Economic Analysis of Four Ciclovia Programs. 
Journal of Urban Health-Bulletin of the New York Academy of Medicine 2012;89(1):153-70. doi: 10.1007/s11524-011-9628-8

70. Moodie M, Haby M, Galvin L, et al. Cost-effectiveness of active transport for primary school children - Walking School Bus program. Int J Behav Nutr Phy 2009;6 doi: Artn 63 10.1186/1479-5868-6-63

71. Verhoef TI, Trend V, Kelly B, et al. Cost-effectiveness analysis of offering free leisure centre memberships to physically inactive members of the public receiving state benefits: a case study. BMC Public Health 2016;16:616. doi: 10.1186/s12889-0163300-x [published Online First: 2016/07/28]

72. Wang G, Macera, C. A., Scudder-Soucie, B., Schimd, T., Pratt, M. \& Buchner, D. A costbenefit analysis of physical activity using bike/pedestrian trails. Health Promotion Practice 2005(6):174-9. 
Table 1: Overview of the modelling approaches used

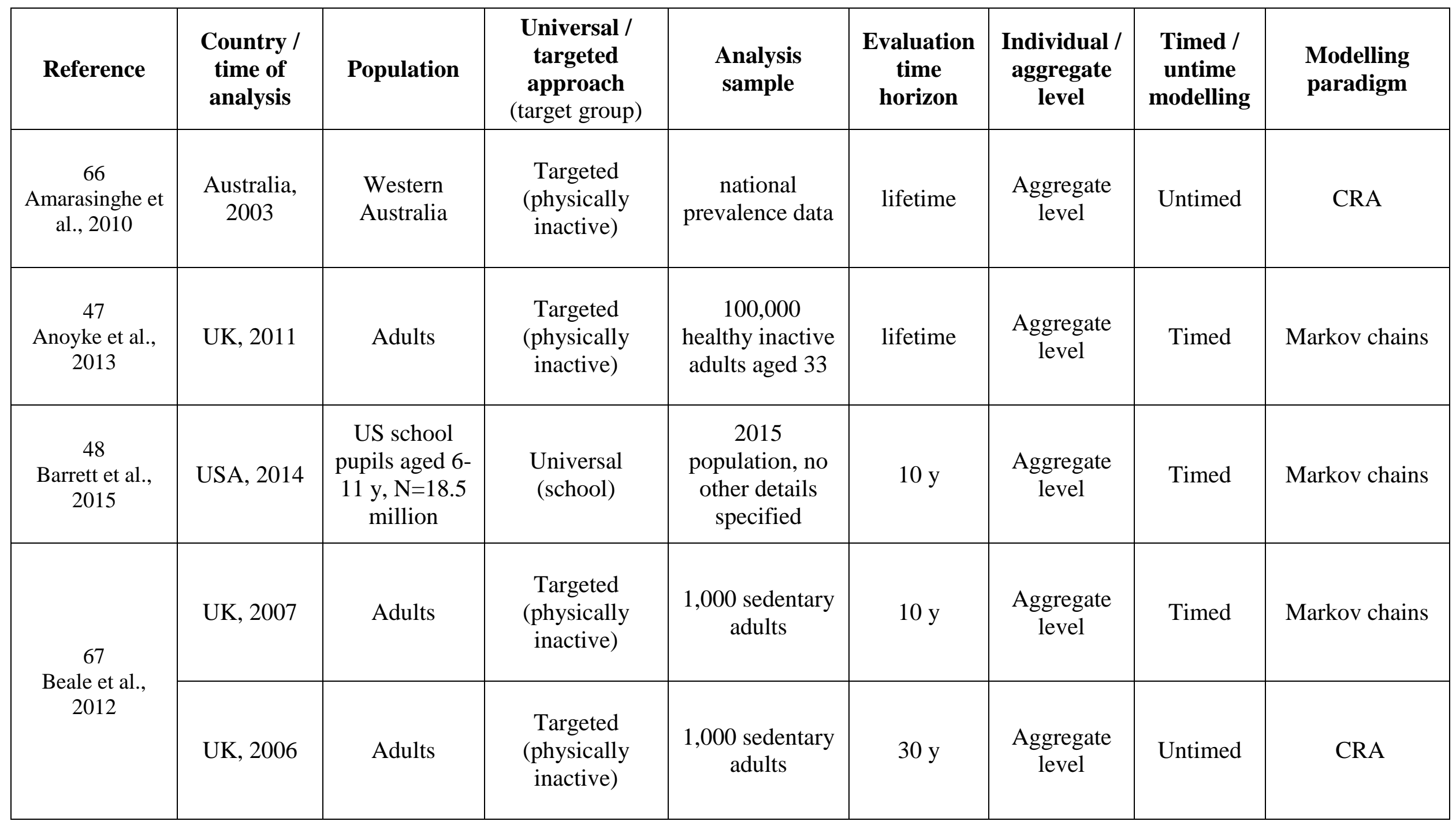




\begin{tabular}{|c|c|c|c|c|c|c|c|c|}
\hline $\begin{array}{c}49 \\
\text { Cavill et al., } \\
2011\end{array}$ & UK, 2009 & not specified & $\begin{array}{l}\text { Universal } \\
\text { (resident } \\
\text { population) }\end{array}$ & $\begin{array}{c}\mathrm{N}=\text { not } \\
\text { specified, } 16 \mathrm{y}+\end{array}$ & $10 y$ & $\begin{array}{c}\text { Aggregate } \\
\text { level }\end{array}$ & Untimed & CRA \\
\hline $\begin{array}{c}36 \\
\text { Cobiac et al., } \\
2009\end{array}$ & $\begin{array}{l}\text { Australia, } \\
2003\end{array}$ & $\begin{array}{l}\text { Australia } \\
\text { population }\end{array}$ & $\begin{array}{c}\text { Simulated } \\
\text { multiple } \\
\text { interventions, } \\
\text { universal and } \\
\text { targeted }\end{array}$ & $\begin{array}{l}\text { Population } \\
\text { age/gender } \\
\text { distribution }\end{array}$ & lifetime & $\begin{array}{c}\text { Aggregate } \\
\text { level }\end{array}$ & Timed & $\begin{array}{l}\text { Multiple cohort } \\
\text { life-table } \\
\text { approach }\end{array}$ \\
\hline $\begin{array}{c}41 \\
\text { Cradock et al., } \\
2017\end{array}$ & USA, 2016 & $\begin{array}{l}\text { School } \\
\text { children }\end{array}$ & $\begin{array}{l}\text { Universal } \\
\text { (school) }\end{array}$ & $\begin{array}{c}2015 \\
\text { population, no } \\
\text { other details } \\
\text { specified }\end{array}$ & $10 y$ & $\begin{array}{c}\text { Individual } \\
\text { level }\end{array}$ & Timed & Microsimulation \\
\hline $\begin{array}{c}68 \\
\text { De Smedt et al., } \\
2012\end{array}$ & $\begin{array}{l}\text { Belgium, } \\
2009\end{array}$ & $\begin{array}{l}\mathrm{N}=245,000 \\
\text { adults } 25-75 \mathrm{y}\end{array}$ & $\begin{array}{l}\text { Universal } \\
\text { (resident } \\
\text { population) }\end{array}$ & $\begin{array}{c}\mathrm{N}=266 \text { adults } \\
25-75 \text { y who } \\
\text { improved PA } \\
\text { level }\end{array}$ & $20 y$ & $\begin{array}{c}\text { Aggregate } \\
\text { level }\end{array}$ & Timed & Markov chains \\
\hline $\begin{array}{c}51 \\
\text { Frew et al., } \\
2012\end{array}$ & UK, 2010 & $\begin{array}{l}\text { City adult } \\
\text { population }\end{array}$ & $\begin{array}{l}\text { Universal } \\
\text { (resident } \\
\text { population) }\end{array}$ & $\begin{array}{c}\mathrm{N}=\text { not } \\
\text { specified, } \\
\text { population of } \\
\text { adults } 16-70 \mathrm{y}, \\
\text { no other details } \\
\text { provided }\end{array}$ & lifetime & $\begin{array}{c}\text { Aggregate } \\
\text { level }\end{array}$ & Timed & Markov chains \\
\hline
\end{tabular}




\begin{tabular}{|c|c|c|c|c|c|c|c|c|}
\hline $\begin{array}{c}39 \\
\text { Goyder et al., } \\
2014\end{array}$ & UK, 2012 & $\begin{array}{l}\text { Sedentary } \\
\text { adults from } \\
\text { deprived } \\
\text { areas }\end{array}$ & $\begin{array}{c}\text { Targeted } \\
\text { (physically } \\
\text { inactive, } \\
\text { middle-aged, } \\
\text { from deprived } \\
\text { socio-economic } \\
\text { backgrounds) }\end{array}$ & $\begin{array}{c}\mathrm{N}=500,000 \\
\text { age/gender } \\
\text { matched } \\
\text { individuals }\end{array}$ & lifetime & $\begin{array}{l}\text { Individual } \\
\text { level }\end{array}$ & Timed & Markov chains \\
\hline $\begin{array}{c}43 \\
\text { Gulliford et al., } \\
2014\end{array}$ & UK, 2010 & $\begin{array}{l}\text { National } \\
\text { population }\end{array}$ & $\begin{array}{l}\text { Targeted } \\
\text { (physically } \\
\text { inactive) }\end{array}$ & $\begin{array}{c}\mathrm{N}=262,704 \\
\text { inactive adults> } \\
30 \mathrm{y}\end{array}$ & 5 and $10 y$ & $\begin{array}{c}\text { Aggregate } \\
\text { level }\end{array}$ & Timed & Markov chains \\
\hline $\begin{array}{c}40 \\
\text { Guo and } \\
\text { Gandavarapu, } \\
2009\end{array}$ & USA, 2001 & $\begin{array}{l}\text { County } \\
\text { population }\end{array}$ & $\begin{array}{l}\text { Universal } \\
\text { (resident } \\
\text { population) }\end{array}$ & $\mathrm{N}=438,881$ & $10 \mathrm{y}$ & $\begin{array}{c}\text { Individual } \\
\text { level }\end{array}$ & Untimed & $\begin{array}{c}\text { System of linear } \\
\text { equations }\end{array}$ \\
\hline $\begin{array}{c}70 \\
\text { Moodie et al., } \\
2009\end{array}$ & $\begin{array}{l}\text { Australia, } \\
2001\end{array}$ & $\begin{array}{l}\text { Primary } \\
\text { school } \\
\text { children }\end{array}$ & $\begin{array}{l}\text { Universal } \\
\text { (school) }\end{array}$ & $\begin{array}{c}\mathrm{N}=15,680 \\
\text { average school } \\
\text { pupils 5-7 y }\end{array}$ & lifetime & $\begin{array}{c}\text { Aggregate } \\
\text { level }\end{array}$ & Untimed & CRA \\
\hline $\begin{array}{c}44 \\
\text { Munro et al., } \\
2004\end{array}$ & UK, 1994 & Older adults & $\begin{array}{l}\text { Universal } \\
\text { (resident } \\
\text { population) }\end{array}$ & $\begin{array}{c}\mathrm{N}=10,000 \\
\text { adults }>=65 \mathrm{y}\end{array}$ & $10 \mathrm{y}$ & $\begin{array}{c}\text { Aggregate } \\
\text { level }\end{array}$ & Untimed & CRA \\
\hline
\end{tabular}




\begin{tabular}{|c|c|c|c|c|c|c|c|c|}
\hline $\begin{array}{c}38 \\
\text { Nshimyumukisa } \\
\text { et al., } 2013\end{array}$ & $\begin{array}{c}\text { Canada, } \\
2008\end{array}$ & Women $>=40 y$ & $\begin{array}{l}\text { Universal } \\
\text { (resident } \\
\text { population) }\end{array}$ & $\begin{array}{c}\mathrm{N}=500,000, \\
\text { women }>=40 \mathrm{y}\end{array}$ & 5 and $10 y$ & $\begin{array}{l}\text { Individual } \\
\text { level }\end{array}$ & Timed & Markov chains \\
\hline $\begin{array}{c}52 \\
\text { Over et al., } \\
2012\end{array}$ & $\begin{array}{c}\text { The } \\
\text { Netherlands, } \\
2009\end{array}$ & $\begin{array}{l}\text { National } \\
\text { population } \\
20-65 \mathrm{y}\end{array}$ & $\begin{array}{l}\text { Targeted } \\
\text { (physically } \\
\text { inactive) }\end{array}$ & $\begin{array}{c}\mathrm{N}=\mathrm{not} \\
\text { specified, } \\
\text { population 20- } \\
65 \mathrm{y}\end{array}$ & lifetime & $\begin{array}{l}\text { Aggregate } \\
\text { level }\end{array}$ & Timed & Markov chains \\
\hline $\begin{array}{c}53 \\
\text { Pringle et al., } \\
2010\end{array}$ & UK, 2006 & Adults & $\begin{array}{c}\text { Simulated } \\
\text { multiple } \\
\text { interventions, } \\
\text { universal and } \\
\text { targeted } \\
\end{array}$ & $\begin{array}{c}\mathrm{N}=\mathrm{not} \\
\text { specified, } \\
\text { population } 10+ \\
\mathrm{y}\end{array}$ & lifetime & $\begin{array}{l}\text { Aggregate } \\
\text { level }\end{array}$ & Untimed & Decision-tree \\
\hline $\begin{array}{c}46 \\
\text { Roux et al., } \\
2008\end{array}$ & USA, 2004 & $\begin{array}{l}\text { Adults } 25-64 \\
\text { years }\end{array}$ & $\begin{array}{c}\text { Simulated } \\
\text { multiple } \\
\text { interventions, } \\
\text { universal and } \\
\text { targeted }\end{array}$ & $\begin{array}{l}\mathrm{N}=\mathrm{not} \\
\text { specified, adult } \\
\text { population } 25- \\
64 \text { years }\end{array}$ & lifetime & $\begin{array}{l}\text { Aggregate } \\
\text { level }\end{array}$ & Timed & Markov chains \\
\hline $\begin{array}{c}42 \\
\text { Gc et al., } 2018\end{array}$ & UK, 2011 & $\begin{array}{c}\text { Adult } \\
\text { population }\end{array}$ & $\begin{array}{l}\text { Universal } \\
\text { (resident } \\
\text { population) }\end{array}$ & $\begin{array}{l}\mathrm{N}=10,000 \\
\text { representative } \\
\text { sample }\end{array}$ & $10 \mathrm{y}$ & $\begin{array}{l}\text { Individual } \\
\text { level }\end{array}$ & Timed & $\begin{array}{l}\text { Discrete event } \\
\text { simulation }\end{array}$ \\
\hline
\end{tabular}




\begin{tabular}{|c|c|c|c|c|c|c|c|}
$\begin{array}{c}71 \\
\begin{array}{c}\text { Verhoef et al., } \\
2009\end{array}\end{array}$ & UK, 2014 & not specified & $\begin{array}{c}\text { Targeted } \\
\text { (physically } \\
\text { inactive) }\end{array}$ & $\begin{array}{c}\mathrm{N}=1025, \\
\text { inactive } \\
\text { adults>18 }\end{array}$ & lifetime & $\begin{array}{c}\text { Aggregate } \\
\text { level }\end{array}$ & Timed \\
$\begin{array}{c}72 \\
\text { Wang et al., } \\
2005\end{array}$ & USA, 1998 & $\begin{array}{c}\text { National } \\
\text { population }\end{array}$ & $\begin{array}{c}\text { Universal } \\
\text { (resident } \\
\text { population) }\end{array}$ & $\begin{array}{c}\mathrm{N}=\mathrm{not} \\
\text { specified, trail } \\
\text { users }\end{array}$ & $30 \mathrm{y}$ & $\begin{array}{c}\text { Aggregate } \\
\text { level }\end{array}$ & Untimed \\
\hline $\begin{array}{c}\text { Zapata-Diomedi } \\
\text { et al., 2017 }\end{array}$ & $\begin{array}{c}\text { Australia, } \\
2013\end{array}$ & $\begin{array}{c}\text { City } \\
\text { population }\end{array}$ & $\begin{array}{c}\text { Universal } \\
\text { (resident } \\
\text { population) }\end{array}$ & $\begin{array}{c}\mathrm{N}=860,000, \\
\text { adult residents }\end{array}$ & lifetime & $\begin{array}{c}\text { Aggregate } \\
\text { level }\end{array}$ & Timed \\
\hline
\end{tabular}

Note: $\mathrm{CRA}=$ comparative risk assessment, $\mathrm{y}=$ years 
Table 2: Critique of the reviewed studies

\begin{tabular}{|c|c|c|c|c|c|c|c|}
\hline \multirow[b]{2}{*}{ Reference } & \multirow[b]{2}{*}{$\begin{array}{c}\text { Modelled } \\
\text { downstream } \\
\text { disease risks }\end{array}$} & \multicolumn{3}{|c|}{ Dynamics of change in PA } & \multirow[b]{2}{*}{$\begin{array}{l}\text { Reflecting } \\
\text { heterogeneity } \\
\text { (details) }\end{array}$} & \multirow[b]{2}{*}{$\begin{array}{c}\text { Incorporation } \\
\text { of health } \\
\text { equity }\end{array}$} & \multirow[b]{2}{*}{$\begin{array}{l}\text { Uncertaint } \\
y \\
\text { assessment }\end{array}$} \\
\hline & & $\begin{array}{c}\text { Natural } \\
\text { course of PA }\end{array}$ & $\begin{array}{c}\text { Time lag to } \\
\text { health } \\
\text { benefit }\end{array}$ & $\begin{array}{c}\text { Decay of } \\
\text { intervention effect } \\
\text { over time }\end{array}$ & & & \\
\hline $\begin{array}{c}66 \\
\text { Amarasinghe et } \\
\text { al., } 2010\end{array}$ & $\begin{array}{c}\text { T2D, } \\
\text { HD,STR,CC,DEP }\end{array}$ & $\begin{array}{l}\text { PA as a fixed } \\
\text { state }\end{array}$ & not specified & $\begin{array}{l}\text { different constant } \\
\text { rates of compliance }\end{array}$ & $\begin{array}{l}\text { average individual, } \\
2 \text { levels of PA }\end{array}$ & not specified & not specified \\
\hline $\begin{array}{c}47 \\
\text { Anoyke et al., } \\
2013\end{array}$ & T2D,CHD,STR & $\begin{array}{l}\text { PA as a fixed } \\
\text { state }\end{array}$ & $\begin{array}{l}1 \text { year run-in } \\
\text { period }\end{array}$ & $\begin{array}{l}100 \% \text { constant, } \\
\text { benefits accrue for the } \\
\text { first } 10 \text { years over } \\
\text { lifetime }\end{array}$ & $\begin{array}{l}\text { average individual, } \\
2 \text { levels of PA }\end{array}$ & not specified & not specified \\
\hline $\begin{array}{c}48 \\
\text { Barrett et al., } \\
2015\end{array}$ & obesity & $\begin{array}{l}\text { PA as a fixed } \\
\text { state }\end{array}$ & $\begin{array}{l}2 \text { years to full } \\
\text { effect on } \\
\text { BMI }\end{array}$ & $\begin{array}{l}100 \% \text { constant, } \\
\text { benefits accrue over } \\
\text { the whole TH }\end{array}$ & not specified & $\begin{array}{c}\text { discussed } \\
\text { implementation } \\
\text { issues potentially } \\
\text { increasing } \\
\text { inequalities }\end{array}$ & not specified \\
\hline \multirow[b]{2}{*}{$\begin{array}{c}67 \\
\text { Beale et al., } \\
2012\end{array}$} & T2D,CHD,STR & $\begin{array}{l}\text { PA as a fixed } \\
\text { state }\end{array}$ & not specified & $\begin{array}{l}100 \% \text { constant, } \\
\text { benefits accrue over } \\
\text { the whole TH }\end{array}$ & $\begin{array}{l}\text { average individual, } \\
2 \text { levels of PA }\end{array}$ & not specified & not specified \\
\hline & not specified & $\begin{array}{l}\text { PA as a fixed } \\
\text { state }\end{array}$ & not specified & $\begin{array}{l}100 \% \text { constant, } \\
\text { benefits accrue over } \\
\text { the whole TH }\end{array}$ & $\begin{array}{l}\text { average individual, } \\
2 \text { levels of PA } \\
\text { (adjustment for age, } \\
\text { gender, ethnicity, } \\
\text { employment status, } \\
\text { education level, } \\
\text { income, marital } \\
\text { status, smoking } \\
\text { status, alcohol }\end{array}$ & not specified & not specified \\
\hline
\end{tabular}




\begin{tabular}{|c|c|c|c|c|c|c|c|}
\hline & & & & & $\begin{array}{l}\text { consumption, and } \\
\text { body mass index) }\end{array}$ & & \\
\hline $\begin{array}{c}49 \\
\text { Cavill et al., } \\
2011\end{array}$ & not specified & $\begin{array}{l}\text { PA as a fixed } \\
\text { state }\end{array}$ & 5 years & $\begin{array}{l}100 \% \text { constant, } \\
\text { benefits accrue over } \\
\text { the whole } \mathrm{TH}\end{array}$ & $\begin{array}{l}\text { average individual, } \\
2 \text { levels of PA } \\
\text { (proportion of } \\
\text { cyclists) }\end{array}$ & not specified & not specified \\
\hline $\begin{array}{c}36 \\
\text { Cobiac et al., } \\
2009\end{array}$ & $\begin{array}{l}\text { T2D, CHD,IHD, } \\
\text { STR, BRC }\end{array}$ & $\begin{array}{l}\text { PA as a fixed } \\
\text { state }\end{array}$ & not specified & $\begin{array}{l}\text { Sustained for the first } \\
\text { year, but decay } \\
\text { exponentially at a rate } \\
\text { of } 50 \% \text { per annum } \\
\text { thereafter }\end{array}$ & $\begin{array}{l}\text { age, gender and } 2 \\
\text { levels of PA }\end{array}$ & not specified & $\begin{array}{l}\text { one-way SA } \\
\text { (dissipation of } \\
\text { effect size) }\end{array}$ \\
\hline $\begin{array}{c}41 \\
\text { Cradock et al., } \\
2017\end{array}$ & obesity & $\begin{array}{l}\text { PA as a fixed } \\
\text { state }\end{array}$ & $\begin{array}{l}\text { not specified } \\
\text { for base-case } \\
\text { analysis, } 2 \text { to } \\
20 \text { years in } \\
\text { SA } \\
\end{array}$ & $\begin{array}{l}100 \% \text { constant, } \\
\text { benefits accrue over } \\
\text { the whole } \mathrm{TH}\end{array}$ & $\begin{array}{c}\text { body mass index, } 2 \\
\text { levels of PA }\end{array}$ & not specified & not specified \\
\hline $\begin{array}{c}50 \\
\text { Dallat et al., } \\
2014\end{array}$ & $\begin{array}{l}\text { T2D, IHD, } \\
\text { STR,CC,BRC }\end{array}$ & $\begin{array}{l}\text { PA as a fixed } \\
\text { state }\end{array}$ & not specified & $\begin{array}{l}100 \% \text { constant, } \\
\text { benefits accrue over } \\
\text { the whole } \mathrm{TH}\end{array}$ & $\begin{array}{l}\text { age, gender and } 2 \\
\text { levels of PA } \\
\text { (walking time) }\end{array}$ & not specified & $\begin{array}{l}\text { one way SA } \\
\text { (time lags) }\end{array}$ \\
\hline $\begin{array}{c}68 \\
\text { De Smedt et al., } \\
2012\end{array}$ & T2D,CHD,STR, CC & $\begin{array}{l}\text { PA as a fixed } \\
\text { state }\end{array}$ & not specified & $\begin{array}{l}100 \% \text { constant, } \\
\text { benefits accrue over } \\
\text { the whole } \mathrm{TH}\end{array}$ & $\begin{array}{l}\text { age, gender, } 3 \text { levels } \\
\text { of PA (national } \\
\text { recommendations) }\end{array}$ & not specified & $\begin{array}{l}50 \% \text { of } \\
\text { intervention } \\
\text { effect after the } \\
\text { first year in } \\
\text { SA }\end{array}$ \\
\hline $\begin{array}{c}51 \\
\text { Frew et al., } 2012\end{array}$ & $\begin{array}{l}\text { T2D, CHD, STR, } \\
\text { CRC, BRC }\end{array}$ & $\begin{array}{l}\text { PA as a fixed } \\
\text { state }\end{array}$ & not specified & $\begin{array}{c}\text { Decline of } 100 \% \text { after } \\
\text { the } 2 \text { years, benefits } \\
\text { accrue over the whole } \\
\text { TH }\end{array}$ & $\begin{array}{l}\text { age, gender and } \\
\text { quintiles of PA }\end{array}$ & $\begin{array}{c}\text { sub-group } \\
\text { analysis is } \\
\text { limited because } \\
\text { of sample size }\end{array}$ & $\begin{array}{l}\text { one-way SA } \\
\text { (dissipation of } \\
\text { intervention } \\
\text { effect on part } \\
\text { of the sample) }\end{array}$ \\
\hline $\begin{array}{l}39 \\
\text { Goyder et al., } \\
2014\end{array}$ & not specified & $\begin{array}{c}\text { PA as a fixed } \\
\text { state }\end{array}$ & not specified & $\begin{array}{l}100 \% \text { constant, } \\
\text { benefits accrue over } \\
\text { the whole } \mathrm{TH}\end{array}$ & $\begin{array}{l}\text { age, gender and PA } \\
\text { (within the "at risk", } \\
\text { three levels) }\end{array}$ & not specified & not specified \\
\hline
\end{tabular}




\begin{tabular}{|c|c|c|c|c|c|c|c|}
\hline $\begin{array}{c}43 \\
\text { Gulliford et al., } \\
2014\end{array}$ & $\begin{array}{c}\text { T2D, CHD, STR, } \\
\text { CRC + DEP } \\
\text { interaction ( } 32 \\
\text { combinations) }\end{array}$ & $\begin{array}{l}\text { PA as a fixed } \\
\text { state }\end{array}$ & not specified & $\begin{array}{l}100 \% \text { constant, } \\
\text { benefits accrue over } \\
\text { the whole } \mathrm{TH}\end{array}$ & $\begin{array}{c}\text { age, gender, } \\
\text { depression status } \\
\text { and } 3 \text { levels of PA } \\
\text { (walking/biking } \\
\text { time) }\end{array}$ & not specified & not specified \\
\hline $\begin{array}{c}40 \\
\text { Guo and } \\
\text { Gandavarapu, } \\
2009\end{array}$ & obesity & $\begin{array}{l}\text { PA as a fixed } \\
\text { state }\end{array}$ & not specified & $\begin{array}{l}100 \% \text { constant, } \\
\text { benefits accrue over } \\
\text { the whole } \mathrm{TH}\end{array}$ & $\begin{array}{l}\text { age, gender, race, } \\
\text { employment status, } \\
2 \text { levels of PA }\end{array}$ & not specified & not specified \\
\hline $\begin{array}{c}69 \\
\text { Montes et al., } \\
2012\end{array}$ & not specified & $\begin{array}{l}\text { PA as a fixed } \\
\text { state }\end{array}$ & not specified & $\begin{array}{c}100 \% \text { constant, } \\
\text { benefits accrue over } \\
\text { the whole } \mathrm{TH}\end{array}$ & $\begin{array}{l}\text { average individual, } \\
2 \text { levels of PA } \\
\text { (proportion of active } \\
\text { commuters to } \\
\text { school) }\end{array}$ & not specified & not specified \\
\hline $\begin{array}{c}70 \\
\text { Moodie et al., } \\
2009\end{array}$ & obesity & $\begin{array}{l}\text { PA as a fixed } \\
\text { state }\end{array}$ & not specified & $\begin{array}{l}100 \% \text { constant, } \\
\text { benefits accrue over } \\
\text { the whole } \mathrm{TH}\end{array}$ & $\begin{array}{l}\text { average individual, } \\
2 \text { levels of PA }\end{array}$ & $\begin{array}{c}\text { discussed } \\
\text { implementation } \\
\text { issues potentially } \\
\text { increasing } \\
\text { inequalities }\end{array}$ & not specified \\
\hline $\begin{array}{l}44 \\
\text { Munro et al., } \\
2004\end{array}$ & not specified & $\begin{array}{l}\text { PA as a fixed } \\
\text { state }\end{array}$ & not specified & $\begin{array}{l}100 \% \text { constant, } \\
\text { benefits accrue over } \\
\text { the whole TH }\end{array}$ & $\begin{array}{l}\text { average individual, } \\
2 \text { levels of PA }\end{array}$ & not specified & not specified \\
\hline $\begin{array}{c}38 \\
\text { Nshimyumukisa } \\
\text { et al., } 2013\end{array}$ & Osteoporosis & $\begin{array}{l}\text { PA as a fixed } \\
\text { state }\end{array}$ & not specified & $\begin{array}{c}\text { explicit constant at } \\
25 \% \text { after } 18 \text { weeks, } \\
\text { benefits accrue over } \\
\text { the whole } \mathrm{TH}\end{array}$ & $\begin{array}{l}\text { age and } 3 \text { levels of } \\
\text { PA (national } \\
\text { recommendations) }\end{array}$ & not specified & not specified \\
\hline $\begin{array}{c}52 \\
\text { Over et al., } 2012\end{array}$ & not specified & $\begin{array}{l}\text { PA as a fixed } \\
\text { state }\end{array}$ & not specified & $\begin{array}{c}50 \% \text { constant, } \\
\text { benefits accrue over } \\
\text { the whole } \mathrm{TH}\end{array}$ & $\begin{array}{l}\text { average individual, } \\
3 \text { levels of PA } \\
\text { (national } \\
\text { recommendations) }\end{array}$ & not specified & not specified \\
\hline
\end{tabular}




\begin{tabular}{|c|c|c|c|c|c|c|c|}
\hline $\begin{array}{l}53 \\
\text { Pringle et al., } \\
2010\end{array}$ & T2D, CHD,STR,CC & $\begin{array}{l}\text { PA as a fixed } \\
\text { state }\end{array}$ & not specified & $\begin{array}{c}\text { explicit } 33 \% \text { to } 50 \% \\
\text { of decline in effect } \\
\text { after year } 2 \text {, benefits } \\
\text { accrue over the whole } \\
\text { TH }\end{array}$ & $\begin{array}{l}\text { age, gender, } 4 \text { levels } \\
\text { of PA - national } \\
\text { recommendations }\end{array}$ & not specified & not specified \\
\hline $\begin{array}{c}46 \\
\text { Roux et al., } \\
2008\end{array}$ & $\begin{array}{l}\text { T2D,CHD,STR, } \\
\text { CRC,BRC }\end{array}$ & $\begin{array}{l}\text { natural history } \\
\text { model }\end{array}$ & not specified & $\begin{array}{c}\text { explicit } 33 \% \text { to } 50 \% \\
\text { of decline in effect } \\
\text { after year } 2 \text {, benefits } \\
\text { accrue over the whole } \\
\text { TH }\end{array}$ & $\begin{array}{l}\text { age, gender and } 4 \\
\text { levels of PA } \\
\text { (national } \\
\text { recommendations) }\end{array}$ & $\begin{array}{c}\text { sub-group } \\
\text { analysis is } \\
\text { limited because } \\
\text { of lack of data }\end{array}$ & $\begin{array}{l}\text { one-way SA } \\
\text { (dissipation of } \\
\text { intervention } \\
\text { effect) }\end{array}$ \\
\hline $\begin{array}{l}45 \\
\text { Roux et al., } \\
2015\end{array}$ & $\begin{array}{l}\text { T2D,CHD,STR, } \\
\text { CRC,BRC }\end{array}$ & $\begin{array}{l}\text { natural history } \\
\text { model }\end{array}$ & not specified & $\begin{array}{l}100 \% \text { constant, } \\
\text { benefits accrue over } \\
\text { the whole } \mathrm{TH}\end{array}$ & $\begin{array}{l}\text { gender and } 4 \text { levels } \\
\text { of PA (national } \\
\text { recommendations) }\end{array}$ & $\begin{array}{c}\text { sub-group } \\
\text { analysis is } \\
\text { limited because } \\
\text { of lack of data }\end{array}$ & $\begin{array}{l}\text { one-way SA } \\
\text { (dissipation of } \\
\text { intervention } \\
\text { effect) }\end{array}$ \\
\hline $\begin{array}{c}42 \\
\text { Gc et al., } 2018\end{array}$ & $\begin{array}{l}\text { T2D, HD,STR,BRC, } \\
\text { LUC,CC, KDC }\end{array}$ & $\begin{array}{l}\text { PA as a fixed } \\
\text { state }\end{array}$ & not specified & $\begin{array}{l}\text { Sustained for the first } \\
\text { year, but decay } \\
\text { exponentially at a rate } \\
\text { of } 55 \% \text { per annum } \\
\text { thereafter }\end{array}$ & $\begin{array}{l}\text { age, sex, ethnicity } \\
\text { and } 3 \text { levels of PA }\end{array}$ & not specified & $\begin{array}{l}\text { one-way SA } \\
\text { (dissipation of } \\
\text { effect size) }\end{array}$ \\
\hline $\begin{array}{c}71 \\
\text { Verhoef et al., } \\
2009\end{array}$ & T2D,CHD,STR & $\begin{array}{l}\text { PA as a fixed } \\
\text { state }\end{array}$ & not specified & $\begin{array}{l}100 \% \text { constant, } \\
\text { benefits accrue over } \\
\text { the whole } \mathrm{TH}\end{array}$ & $\begin{array}{l}\text { average individual } \\
\text { and } 3 \text { levels of PA } \\
\text { (national } \\
\text { recommendations) }\end{array}$ & not specified & not specified \\
\hline $\begin{array}{c}72 \\
\text { Wang et al., } \\
2005\end{array}$ & not specified & $\begin{array}{l}\text { PA as a fixed } \\
\text { state }\end{array}$ & not specified & $\begin{array}{l}100 \% \text { constant, } \\
\text { benefits accrue over } \\
\text { the whole } \mathrm{TH}\end{array}$ & $\begin{array}{l}\text { average individual, } \\
2 \text { levels of PA }\end{array}$ & not specified & not specified \\
\hline $\begin{array}{c}37 \\
\text { Zapata-Diomedi } \\
\text { et al., } 2017\end{array}$ & $\begin{array}{c}\text { T2D, } \\
\text { IHD,STR,CC,BRC }\end{array}$ & $\begin{array}{l}\text { PA as a fixed } \\
\text { state }\end{array}$ & not specified & $\begin{array}{l}\text { gradual increase, } \\
\text { benefits accrue over } \\
\text { the whole } \mathrm{TH}\end{array}$ & $\begin{array}{c}\text { Household-level: } \\
\text { age, gender, } \\
\text { geographical } \\
\text { location and } 2 \text { levels } \\
\text { of PA }\end{array}$ & not specified & not specified \\
\hline
\end{tabular}

Note: $\mathrm{BRC}=$ breast cancer, $\mathrm{CC}=$ colon cancer, $\mathrm{CHD}=$ coronary heart disease, $\mathrm{DEP}=$ depression, $\mathrm{CRC}=$ colorectal cancer, $\mathrm{HD}=$ heart disease, $\mathrm{KDC}=$ kidney cancer, $\mathrm{IHD}=$ ischaemic heart disease, $\mathrm{LUC}=$ lung cancer, $\mathrm{PA}=$ physical activity, $\mathrm{SA}=$ sensitivity analysis, $\mathrm{STR}=$ stroke, $\mathrm{T} 2 \mathrm{D}=$ type $\mathrm{II}$ diabetes, $\mathrm{TH}=$ time horizon, $\mathrm{y}=\mathrm{years}$. 


\section{Figure 1 PRISMA flow diagram of the literature search and selection process}

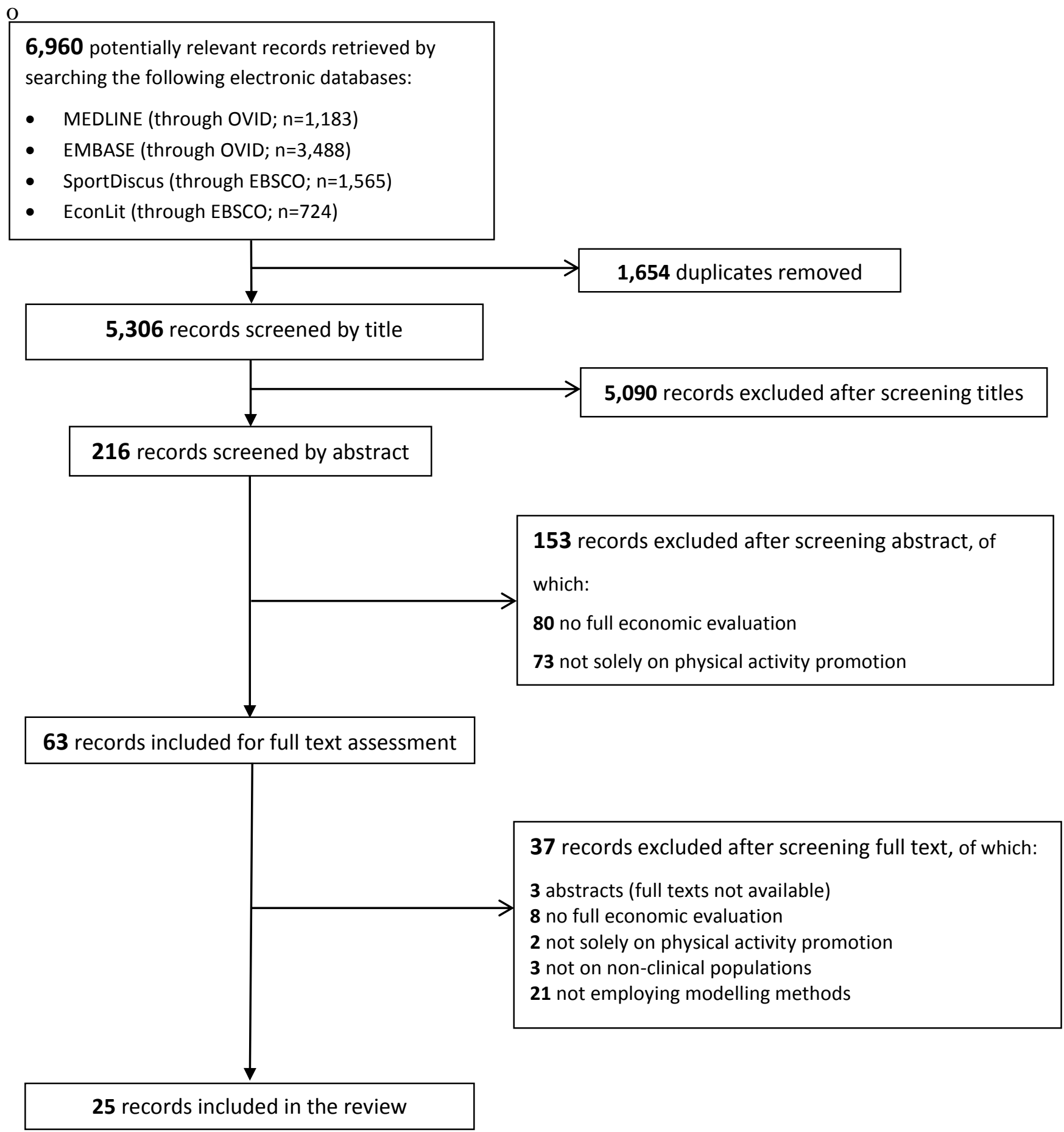


Revision Notes (without Author details)

\begin{tabular}{|c|c|c|}
\hline \multirow[t]{2}{*}{ Reviewer: 1} & \multicolumn{2}{|l|}{ Comment } \\
\hline & $\begin{array}{l}\text { * None of the studies accounted for natural fluctuation in PA levels over time. I agree that the PA models should consider or at least discuss this } \\
\text { issue. However, whether to include this in the model also depends on factors such as time horizon of the analysis, model cycle length (e.g. } \\
\text { monthly vs annual), and study population (e.g. young adults). Incorporating this into models to evaluate PA intervention in adults that are known } \\
\text { to have stable PA habits and with a short time horizon would additionally add complexity. This aspect is not well reflected in the discussion. }\end{array}$ & $\begin{array}{l}\text { Answer: thanks for this comment. The Discussion section has } \\
\text { been edited to include "However, in scenarios where PA levels are } \\
\text { likely to be stable, the decision to incorporate this element into the } \\
\text { model needs to be evaluated in light of the trade-off between added } \\
\text { complexity and accuracy." }\end{array}$ \\
\hline & ${ }^{*}$ Page 6 , line 29 - please spell out NICE. & Answer: NICE spelt out \\
\hline & * There are still issues with referencing. The main text cites references up to $\# 65$, but the reference list includes 72 papers. & $\begin{array}{l}\text { Answer: The reason for this is that not all of the } 25 \text { reviewed studies } \\
\text { are directly cited within the manuscript, so the difference in } 7 \\
\text { references is given by those studies. }\end{array}$ \\
\hline & $\begin{array}{l}\text { *Table } 1 \text { - it is hard to link the reference \# with the study in column } 1 \text {. Suggest adding author name, i.e. Amarasinghe et al66 to improve } \\
\text { readability. Did the authors of ref\# } \# 7 \text { use two times of analysis, i.e. } 2007 \text { and } 2006 \text { ? }\end{array}$ & $\begin{array}{l}\text { Answer: Added authors' name to reference numbers to improved } \\
\text { readibility in both Table } 1 \text { and } 2 \text {. Yes, the authors of ref } \# 67 \text { (Beale et } \\
\text { al., 2012) use two times of analysis, namely, } 2007 \text { and } 2006 .\end{array}$ \\
\hline & "There is inconsistent use of country names (United Kingdom, UK, US and USA). Please use them consistently throughout the paper. & Answer: corrected to UK and USA \\
\hline & $\begin{array}{l}\text { " Figure } 1 \text { - total number of studies for two boxes ( } n=153 \text { and } n=37 \text { ) do not match. Suggest replacing EE with economic evaluation. Check all } \\
\text { acronyms and remove if not using them more than five times in the paper. }\end{array}$ & $\begin{array}{l}\text { Answer: Total number of studies for the } \mathrm{n}=153 \text { and } \mathrm{n}=37 \text { boxes } \\
\text { corrected. Replaced all acronyms, including } \mathrm{EE} \text {, with their } \\
\text { corresponding full words. }\end{array}$ \\
\hline & * Table 2, column 2 heading - what does 'other' refer in 'Modelled downstream disease risks & Answer: "/other" removed \\
\hline
\end{tabular}


Modelling the impact of physical activity on public health: a review and critique 
Background While several reviews have assessed economic evaluations of physical activity in public health and, in most cases, found the interventions to be cost-effective, the validity of the conclusions reached depends on the appropriateness of the modelling methods used in the individual studies.

Objective To provide an overview and critique of modelling approaches and key structural assumptions used in applied studies to estimate the impact of physical activity on health. Methods Electronic databases were systematically searched for relevant model-based economic evaluations. A thematic approach was used to assess the modelling studies. The critique determined the appropriateness of the modelling frameworks and plausibility of key structural assumptions.

Results Twenty-five models were identified. Cohort models were most frequently used. High variability in the modelling of downstream diseases was found across studies analysing similar populations. Structural assumptions regarding the dynamics of change of physical activity were unrealistic in most cases. Heterogeneity was addressed in only a few studies, while health equity concerns were, at best, acknowledged by authors.

Conclusions This literature is predominantly characterised by modelling approaches that may not adequately address the complexities associated with representing the physical activity behaviour- population health process. A consensus on how to model the impact of physical activity on public health and development of a reference model could help reduce these sources of uncertainty.

Keywords: physical activity, economic evaluation, modelling, public health. 


\section{INTRODUCTION}

The finite resources available to decision makers dictates that commissioning of interventions ought to be based not only on the grounds of effectiveness, but also on cost-effectiveness ${ }^{1}$. To support reimbursement decisions concerned with funding interventions where there are multiple options, economic evaluation is typically used ${ }^{2}$.

Several studies have reviewed the cost-effectiveness evidence for promoting physical activity (PA) in the general population and found the interventions to provide value for money in most cases $^{3-7}$. However, methodological reviews have highlighted a number of challenges related to the economic evaluation of public health interventions, including $\mathrm{PA}^{8-11}$. Covering all elements of the evaluation, from research design of the intervention to the statistical and economic analyses, these challenges have previously been grouped into four broad categories: attribution of effects, measuring and valuing outcomes, intersectoral costs and consequences and incorporating equity concerns.

A recent review examined how the four methodological challenges above have been addressed in applied studies, across two categories of PA interventions (targeted PA and sedentary behaviour $)^{11}$. Confirming previous findings ${ }^{8-10}$, this review found an overall poor quality of reporting and marked inconsistencies in the methods applied across economic evaluations, providing a series of recommendations for the design, analysis and appraisal of economic evaluations.

The existing methodological reviews did not provide a critique on the appropriateness of the modelling approaches used to estimate the impact of changes in PA on population health. More specifically, they did not comment on the appropriateness of structural features of the modelling approach and if these aligned with the fundamental nature of the behaviourpopulation health process they were designed to represent ${ }^{12,13}$. This is important as the lack 
of adequate structure and unrealistic model assumptions can hinder the validity of costeffectiveness findings ${ }^{14}$.

\section{Challenges in modelling the impact of physical activity on public health}

More specifically, in terms of measuring effectiveness, a number of challenges in modelling healthy behaviours for public health economic evaluations, including PA, have been discussed in the literature ${ }^{14,16}$. These challenges derive from key complexities of the behaviour - population health process, which require the use of form of modelling, and include: 1) the link to multiple chronic diseases; 2) a dynamic nature of behaviour and 3) heterogeneous response to the intervention.

A recent scientific report ${ }^{17}$, which forms the basis of the current UK PA guidelines ${ }^{18}$, describes the relationship between PA and the downstream risk of disease, by assessing the relevant available evidence from systematic reviews and meta-analysis against five criteria (i.e. applicability, generalisability, risk of bias or study limitations, quantity and consistency and magnitude and precision of effect). This report confirmed that there was strong evidence suggesting a link between PA and metabolic (e.g. type II diabetes), cardiovascular (e.g. coronary heart disease and stroke), genetic mutation (e.g. colorectal and breast cancer), mental (e.g. depression) and geriatric (e.g. falls) conditions. In addition, disease probabilities may not be independent from one another. For instance, the risk of type II diabetes has been associated with risk of colorectal and breast cancers, mainly due to shared risk factors, among which PA plays a major role ${ }^{19}$.

From a modelling standpoint, this complexity represents a technical problem in that a heathy individual faces a number of different competing and complementary disease risks at any given time, based on the individuals' PA level and other personal characteristics.

Furthermore, some disease risks which will be affected by changes in PA habits in the short 
term (e.g. psychological benefits ${ }^{20}$ ), while others will take some time before occurring (e.g. colorectal cancer incidence $\left.{ }^{21}\right)$.

While PA habits generally tend to be stable over adulthood ${ }^{22}$, a decline in PA is typically associated with older age ${ }^{23}$. Evidence has also shown that natural fluctuations in PA can occur due to seasonality effects or particular life phases ${ }^{24}$. Furthermore, it is also reasonable to expect that different individuals not only will react heterogeneously, in terms of change in behaviour to the same level of exposure, but also that behaviour changes will be sustained at different rates over time ${ }^{25}$. Although the magnitude of effect will depend on the type of intervention, assuming that the changes induced by the intervention over the short term will remain constant over time is likely to be unrealistic, whereas rebound trajectories are generally more likely to occur ${ }^{26}$.

Finally, it is important to take into account heterogeneity in natural history of the PA behaviour-health process and intervention effects on this process for two main reasons: to reduce the risk of inducing bias in the cost-effectiveness results and to align the modelling approach to the objectives of the decision-makers the model is aimed to inform ${ }^{2,12}$. Public health decision-makers have a prominent goal of reducing existing health disparities in the population, for instance associated with socio-economic factors ${ }^{27}$, reflecting the value that society places on lessening unfair inequalities alongside improving health ${ }^{28,29}$.

\section{Modelling frameworks available to analysts}

There has been a wealth of research dedicated to generating taxonomies of the mathematical / epidemiological paradigms available to health economic modellers ${ }^{30-32}$. This has also reflected on their properties to inform model-based economic evaluations in public health. These frameworks have been broadly categorised into cohort and individual level approaches 
and based on their ability to formally capture time-dependent effects and interactions between individuals and the environment ${ }^{32}$.

Briefly, cohort-level approaches are generally simpler than individual level frameworks. In ascending order of modelling capacity, with decision trees and comparative risks assessments (CRAs) neither time nor interactions can be explicitly considered. Markov chains, which can be implemented at both an individual and a cohort level, can instead represent time in the process explicitly as a sequence of transitions between states. More complex are discrete time events and agent-based models which, despite their ability to formally represent changes in states over time and interactions between individuals (the latter), using either discrete and continuous time frameworks, have seen limited application in public health ${ }^{32}$.

Beside the suitability of the modelling framework, the way these methods are implemented in practice (i.e. what structural assumptions are made) can affect the validity of costeffectiveness findings ${ }^{15}$. Comparably to how the National Institute of Health and Care Excellence (NICE)NHCE evaluates models included as part of manufacturers submissions ${ }^{33}$, previews attempts to critique the plausibility of key structural assumptions of the models have been made in other public health evaluation settings ${ }^{10,16}$. To date, no methodological review has focussed on investigating these issues within the PA literature. The present paper aims to fill this gap.

\section{METHODS}

Details regarding the search strategy, eligibility criteria, study screening and selection methods are described in Appendix I. In brief, model-based economic evaluations of PA interventions were identified within the published literature, from database inception to April 2019. Given the review focus, only full economic evaluations were included (i.e. cost-utility, 
cost-benefit, cost-consequences and cost-benefit analyses). A literature search of four electronic databases (MEDLINE, EMBASE, SportDiscus and EconLit) was conducted, with free-text terms, synonyms, spelling variants, abbreviations and indexing terms related to the three concepts of economic evaluation, model and physical activity being used to identify relevant articles.

Data extraction forms were developed by adapting existing templates suggested by review guides ${ }^{34,35}$. These forms were designed to extract information on what modelling frameworks were applied and what key structural assumptions of the models relating to the three complexities discussed above (i.e. the link of physical inactivity to multiple chronic diseases; a dynamic nature of PA behaviour and heterogeneous response to the intervention) were made for estimation of the intervention impact on population health. As recommended for methodological reviews ${ }^{34,35}$, methods were summarised using a thematic approach. For reference, an overview of the modelling studies is first presented. This includes details on the decision contexts and mathematical frameworks used for economic evaluation, based on a classification recently suggested ${ }^{32}=$ The second part of the review critiqued the base-case structural assumptions of the models in terms of their plausibility against the existing available evidence. While these assumptions are often interrelated, an evaluation of how the related complexities have been handled in practice is presented. The following questions thus formed the basis of the extraction process, under four themes:

- $\quad$ Modelled downstream disease risk:

$\quad$ what and how diseases associated with PA have been modelled?

- Dynamic nature of PA:

○ does the model allow for natural fluctuations in PA levels over time?; 
o what assumptions have been made with regard to the link between exposure and change in PA / gains in health?;

- what assumptions have been made with regard to time-dependency of intervention effects?;

- $\quad$ Reflecting heterogeneity:

o how differences in intervention impacts between individual characteristics have been reflected?

- Incorporating health equity concerns:

○ how intervention impacts on existing health inequalities have been modelled?

\section{RESULTS}

\section{Included studies}

Figure 1 shows a PRISMA flow diagram of the literature search and selection process.

Twenty-five papers met the selection criteria. Table 1 provides an overview of the modelling studies. Eleven papers based their analyses on primary data from the United KingdomUK, seven from the USA, four from Australia and one each from Belgium, Canada and The Netherlands. The majority of studies focused on adults ( $>=18$ years, $n=20$ ), four analyses focused only school pupils, and two included a mixed population (adults and children). Interventions based on universal approaches to promotion were explored in the majority of studies $(18 / 25)$.

\section{Modelling frameworks}

Eighty percent of the studies $(n=20)$ employed aggregate-level approaches, nine of which used untimed modelling methods (eight CRAs and one decision-tree). Eleven analyses were 
based on discrete-time frameworks, with two multiple cohort lifetable approaches ${ }^{36,37}$, and Markov chain modelling being used the most frequently. Of the five individual-level models, two were Markov chains ${ }^{38,39}$, one applied a system of linear equations using a cross-sectional regression analysis approach ${ }^{40}$, one a microsimulation approach ${ }^{41}$ (although no details were reported in terms of Markovian assumptions or interaction-levels) and one study developed a discrete event simulation model ${ }^{42}$.

\section{Modelling of downstream disease risk}

Table 2 summarises the key structural assumptions made in order to estimate the impact of changes in PA on public health. The majority of studies $(n=23)$ evaluated the impact of interventions on chronic diseases and conditions associated with PA, with eight of these studies not stating which diseases were considered. The number of chronic diseases ranged from one to seven, with one study modelling 32 disease combinations ${ }^{43}$.

Except for one study which focused on Osteoporosis ${ }^{38}$, all the models selected Type II diabetes and at least one cardiovascular disease (either a type of Stroke or Coronary Heart Disease). Eleven models included at least one cancer (i.e. Colon, Colorectal, Breast, Lung and Kidney) and only two studies considered impacts on mental health outcomes, specifically, depression. Only one study ${ }^{44}$ included exercise-related injuries among the consequences. For those studies focussing on adults from the general population $(n=14)$, the majority $(\mathrm{n}=8)$ selected five chronic conditions. In addition, while the models evolved over time in terms of disease selection, except for one study which applied random search method to calibrate disease risk parameters ${ }^{42}$, none of the models took into account synergistic and compensatory effects between disease risks.

Thus, none of the reviewed models would be able to reflect the currently available evidence on the associated chronic disease risk, therefore, not adequately capturing the impact of 
changes in PA, either due to a narrow evaluation scope and/or lack of adjustments for interaction between diseases.

\title{
Dynamics of physical activity
}

\author{
Natural course of PA \\ Except for two studies that developed a natural history model from PA available from a \\ country-level surveillance system ${ }^{45} 46$, none of the models accounted for natural fluctuations \\ in PA levels over time. Baseline PA states were assumed to be stable, also when long-term \\ time horizons were considered, 15/25 evaluations with time horizons equal to or longer than \\ 30 years). Furthermore, transitions between the highest and the lowest levels of PA were not \\ allowed.
}

\section{Link between exposure and response and its time dependency}

All but four models reported that changes in PA and related health gains would be immediate following exposure to the intervention. One study ${ }^{47}$ assumed that the intervention could not affect disease risk in the first year ("run-in period"). Other analyses assumed that it would take two years ${ }^{48}$, or up to five years ${ }^{49}$, for the intervention to reach full effect. Except for one report which reported on the time lag assumed between changes in PA and disease occurrence used in the model ${ }^{41}$, none of the other studies provides details regarding time lag to disease.

\section{Time-dependent intervention effects}

The majority of models (15/25) assumed implicitly or explicitly that the intervention effect would not decay after the intervention ended (i.e. beyond follow-up assessment period). The remaining 10 analyses assumed a constant and homogeneous decline in effect, ranging from $25 \%$ to $100 \%$, up to two years after the intervention ended. 
Although lack of data may have driven these choices, the structural assumptions related to the dynamics of change in PA that underlie the current models are underreported and may be unrealistic. While appropriate sensitivity analysis may have helped characterise these complexities for decision-making ${ }^{2}$, only a minority of models explored the impact of variations to these base-case structural assumptions (see Table 2).

\section{Reflecting heterogeneity}

Ten studies used population average approaches, evaluating the health impact of changes in PA levels in homogeneous groups of inactive / sedentary adults or school pupils. Baseline differences in PA were considered in only nine studies. Three to five levels (i.e. PA states) were defined in these analyses, with part of the models aligning the classification of PA levels to current national-level PA recommendations ${ }^{37,45,46,51-53}$. Twelve studies accounted for heterogeneous health impacts based on at least age or gender, with two analyses also considering ethnicity/race differences ${ }^{40,42}$.

Thus, while some degree of heterogeneity has been captured by the reviewed models, only a minority addressed this aspect formally by considering at least basic socio-demographic differences, and in particular, baseline PA level.

\section{Incorporating health equity}

None of the reviewed economic models incorporated concerns relating to the distributional impact of the intervention formally into the economic evaluation. Where models accounted for heterogeneous effects, they did not report subgroup analysis which would have allowed for inferences about effects on health inequalities. In only a minority of studies (5/25) equity considerations were discussed. 


\section{DISCUSSION AND CONCLUSIONS}

\section{Main findings}

Complementary to previous reviews ${ }^{8-11,14}$, this is the first methodological review to provide an overview and critique of the modelling approaches used in model-based economic evaluations for estimating impacts of changes in PA on public health. This review has shed light on key structural assumptions that underlie the models, which can aid interpretation of the cost-effectiveness findings and highlights model development opportunities.

If, on one hand, economic evaluation guidelines support the notion that model structures should be kept as simple as possible $e^{54,55}$, models should align to the nature of the process they are intended to represent ${ }^{12}$. While acknowledging that the trade-off between simplicity and internal validity still represents an unresolved challenge for modellers, this literature is predominantly characterised by modelling approaches that may not adequately address the complexities associated with the PA behaviour - population health process they were intended to represent. In addition, key structural assumptions were often unsupported by relevant evidence and based on general rules of thumb.

\section{Minimum modelling standards}

Based on the findings from this review, there are a number of areas in which minimum modelling standards should be established. First, modelling of downstream disease risks. The disease selection in the reviewed models does not reflect the current available epidemiological evidence on the relationship between PA and chronic disease. While differences in environmental-level factors may explain differences in magnitude of risk between settings (e.g. countries), disease selection for identified populations should be only based on strong and updated epidemiological evidence. Disease risks are not likely to be 
independent from one another, and this should be taken into account, either formally (e.g. using calibration methods) or informally (e.g. by means of scenario analysis).

Second, accommodating for the dynamics of PA. Natural trends in PA levels should be used as baseline data for comparison. Conceptualising PA levels as fixed states is likely to induce bias in the estimations, especially when modelling PA behaviours that are subject to seasonality effects (e.g. gym attendance in summer) or over sensitive life phases (e.g. retirement). However, in scenarios where PA levels are likely to be stable, the decision to incorporate this element into the model needs to be evaluated in light of the trade-off between added complexity and accuracy. Assumptions regarding time lags and decay of intervention effects over time must also be made explicit. The current lack of data on long-term impacts of PA intervention does not justify the use of unrealistic assumptions. Instead, modelling of PA should include analysis of a range of possible scenarios for adequately reflecting the uncertainty surrounding the decision ${ }^{2}$. The common assumption found across the models of no decay of effect over time is unrealistic, especially when assessing effects over long periods of time. This is likely to result in overestimation of the health benefits of the intervention and undermine the validity of research findings.

Third, reflecting heterogeneity. Models should capture systematic differences in impacts between individual characteristics ${ }^{56}$. Moderator variables should be identified both for the estimation of effectiveness and cost-effectiveness of PA interventions directed at heterogeneous populations. Although sub-group analyses will not always be feasible in practice (e.g. due to small sample sizes), reports should make explicit the reasons for not conducting them. Reports should also include details regarding the gradients in risks between population sub-groups, and how the shape of these gradients is assumed (e.g. curvilinear ${ }^{57}$ ) when no data were available. 
Forth, on the issue of incorporating health inequity concerns. Methods for incorporating such

\section{Limitations}

This review is subject to several limitations. The level of detail presented in the applied studies leads us to conclude that an overall poor quality of reporting was observed, making the assessment of the modelling studies difficult. However, a formal quality assessment was not conducted according to previously defined criteria, limiting the ability of this review to determine a more traditional judgement on study quality. In addition, whilst a detailed systematic search was conducted, this review only included PA interventions, therefore 
limiting the generalisability of the findings to economic evaluations of interventions where PA is combined with other technologies or healthy behaviours.

This study focused on the cost-effectiveness literature only, and it is possible that other types of studies, such as epidemiological studies, have addressed some of the complexities discussed. Furthermore, methodological aspects discussed here represent only a proportion of the challenges that are apparent when modelling of impact of different types of PA interventions. Specifically, modelling issues related to compensatory or synergistic effects with other behaviours on the pathway to health improvement, such as dietary patterns, interactions between individuals, and non-health effects of these interventions, have not been addressed in this review and should be the focus of further research.

\section{Concluding remarks}

Reaching a consensus on minimum modelling standards, improved reporting of the key model assumptions using the available reporting checklists ${ }^{61-64}$ and development of a reference model $^{65}$ could help reduce these sources of uncertainty, and therefore support optimal decision-making. 


\section{REFERENCES}

1. National Institute for Health and Care Excellence. Methods for the development of NICE public health guidance (third edition) 2012 [Available from:

https://www.nice.org.uk/process/pmg4/resources/methods-for-the-development-ofnice-public-health-guidance-third-edition-pdf-2007967445701.

2. Briggs A, Sculpher, M., Claxton, K.,. Decision Modelling for Health Economic Evaluation. Oxford: Oxford University Press 2006.

3. Garrett S, Elley CR, Rose SB, et al. Are physical activity interventions in primary care and the community cost-effective? A systematic review of the evidence. Br J Gen Pract 2011;61(584):e125-33. doi: 10.3399/bjgp11X561249 [published Online First: 2011/03/08]

4. Gc V, Wilson EC, Suhrcke M, et al. Are brief interventions to increase physical activity cost-effective? A systematic review. Br J Sports Med 2016;50(7):408-17. doi: 10.1136/bjsports-2015-094655 [published Online First: 2015/10/07]

5. Gordon L, Graves N, Hawkes A, et al. A review of the cost-effectiveness of face-to-face behavioural interventions for smoking, physical activity, diet and alcohol. Chronic Illn 2007;3(2):101-29. doi: 10.1177/1742395307081732 [published Online First: 2007/12/18]

6. Laine J, Kuvaja-Kollner V, Pietila E, et al. Cost-effectiveness of population-level physical activity interventions: a systematic review. Am J Health Promot 2014;29(2):71-80. doi: 10.4278/ajhp.131210-LIT-622 [published Online First: 2014/11/02]

7. Pavey TG, Anokye N, Taylor AH, et al. The clinical effectiveness and cost-effectiveness of exercise referral schemes: a systematic review and economic evaluation. Health Technol Assess 2011;15(44):i-xii, 1-254. doi: 10.3310/hta15440 [published Online First: 2011/12/21]

8. Weatherly H, Drummond M, Claxton K, et al. Methods for assessing the cost-effectiveness of public health interventions: key challenges and recommendations. Health Policy 2009;93(2-3):85-92. doi: 10.1016/j.healthpol.2009.07.012 [published Online First: $2009 / 08 / 28]$

9. Alayli-Goebbels AF, Evers SM, Alexeeva D, et al. A review of economic evaluations of behavior change interventions: setting an agenda for research methods and practice. J 
Public Health (Oxf) 2014;36(2):336-44. doi: 10.1093/pubmed/fdt080 [published Online First: 2013/08/24]

10. Griffiths UK, Anigbogu B, Nanchahal K. Economic evaluations of adult weight management interventions: a systematic literature review focusing on methods used for determining health impacts. Appl Health Econ Health Policy 2012;10(3):145-62. doi: 10.2165/11599250-000000000-00000 [published Online First: 2012/03/24]

11. Cochrane M, Watson PM, Timpson H, et al. Systematic review of the methods used in economic evaluations of targeted physical activity and sedentary behaviour interventions. Soc Sci Med. 2019;232:156-167. doi:10.1016/j.socscimed.2019.04.040

12. Squires H, Chilcott J, Akehurst R, Burr J, Kelly MP. A Framework for Developing the Structure of Public Health Economic Models. Value Health. 2016;19(5):588-601. doi:10.1016/j.jval.2016.02.011

13. Candio, P. 2019. Economic evaluation of universal programmes to promote healthy behaviours: challenges and possible solutions with an application to physical activity (Doctoral thesis).

[online :http://etheses.whiterose.ac.uk/26439/1/PhD\%20thesis\%20document\%20$\% 20$ candio.pdf]

14. Squires H, Chilcott J, Akehurst R, Burr J, Kelly MP. A systematic literature review of the key challenges for developing the structure of public health economic models. Int $\mathrm{J}$ Public Health. 2016;61(3):289-298. doi:10.1007/s00038-015-0775-7

15. Tsoi B, Jegathisawaran J, Tarride J-E, Blackhouse G, O’Reilly D (2015) Do different decision-analytic modelling approaches produce different cost-effectiveness results? Insights from a systematic review of existing cross-validation studies. Expert Rev Pharmacoecon Outcomes Res 15(3):451-463

16. Tsoi B, O'Reilly D, Jegathisawaran J, Tarride JE, Blackhouse G, Goeree R. Systematic narrative review of decision frameworks to select the appropriate modelling approaches for health economic evaluations. BMC Res Notes. 2015;8:244. Published 2015 Jun 17. doi:10.1186/s13104-015-1202-0

17. Office of Disease Prevention and Health Promotion. Physical Activity Guidelines Advisory Committee Scientific Report 2018 [Available from: https://health.gov/paguidelines/secondedition/report/pdf/04_C_Background_and_Key_Physical_Activity_Concepts.pdf. 
18. Chief Medical Officers. UK Chief Medical Officers' Physical Activity Guidelines 2019 [Available from:

https://assets.publishing.service.gov.uk/government/uploads/system/uploads/attachme nt_data/file/832868/uk-chief-medical-officers-physical-activity-guidelines.pdf.

19. Giovannucci E, Harlan DM, Archer MC, et al. Diabetes and Cancer: A Consensus Report. Ca-Cancer J Clin 2010;60(4):207-21. doi: 10.3322/caac.20078

20. Mandolesi L, Polverino A, Montuori S, et al. Effects of Physical Exercise on Cognitive Functioning and Wellbeing: Biological and Psychological Benefits. Front Psychol 2018;9 doi: ARTN 509 10.3389/fpsyg.2018.00509

21. Oruc Z, Kaplan MA. Effect of exercise on colorectal cancer prevention and treatment. World J Gastro Oncol 2019;11(5):348-66. doi: 10.4251/wjgo.v11.i5.348

22. Cleland V, Dwyer T, Venn A Which domains of childhood physical activity predict physical activity in adulthood? A 20-year prospective tracking study British Journal of Sports Medicine 2012;46:595-602.

23. Westerterp KR. Changes in physical activity over the lifespan: impact on body composition and sarcopenic obesity. Obes Rev 2018;19 Suppl 1:8-13. doi: 10.1111/obr.12781 [published Online First: 2018/12/05]

24. Tucker P, Gilliland J. The effect of season and weather on physical activity: a systematic review. Public Health 2007;121(12):909-22. doi: 10.1016/j.puhe.2007.04.009 [published Online First: 2007/10/09]

25. Van Dyck D, Mertens L, Cardon G, et al. Opinions Toward Physical Activity, Sedentary Behavior, and Interventions to Stimulate Active Living During Early Retirement: A Qualitative Study in Recently Retired Adults. J Aging Phys Act 2017;25(2):277-86. doi: 10.1123/japa.2015-0295 [published Online First: 2016/10/21]

26. Neil Howlett, Daksha Trivedi, Nicholas A Troop, Angel Marie Chater, Are physical activity interventions for healthy inactive adults effective in promoting behavior change and maintenance, and which behavior change techniques are effective? A systematic review and meta-analysis, Translational Behavioral Medicine, Volume 9, Issue 1, February 2019, Pages 147-157, https://doi.org/10.1093/tbm/iby010

27. Marmot M. Fair Society, Healthy Lives The Marmot Review 2010 [Available from: https://www.parliament.uk/documents/fair-society-healthy-lives-full-report.pdf. 28. Exworthy, M, Oliver, M. Evidence and health inequalities: the Black, Acheson and Marmot reports. In: Exworthy, M, Peckham, S, Powell, M, Hann, A, eds. Shaping Health Policy Case Study Methods and Analysis. Bristol, UK: Policy Press; 2012. 
29. Guindo, LA, Wagner, M, Baltussen, R. From efficacy to equity: literature review of decision criteria for resource allocation and healthcare decisionmaking. Cost Effectiveness Resource Allocation. 2012;10(1):9.

30. Brennan A, Chick SE, Davies R (2006) A taxonomy of model structures for economic evaluation of health technologies. Health Econ 15:1295-1310

31. Chick SE (2007) Taxonomy of model structure for health economics. http://www2.wmin.ac.uk/hscmg/qmmhealth2007/talks/Chick_S.IMAHealth2007..Keynote.pdf. Accessed 20 Jan 2014

32. Briggs AD, Wolstenholme J, Blakely T, et al. Choosing an epidemiological model structure for the economic evaluation of non-communicable disease public health interventions. Popul Health Metr 2016;14:17. doi: 10.1186/s12963-016-0085-1 [published Online First: 2016/05/07]

33. National Institute for Health and Care Excellence. Guide to the processes of technology appraisal, 2018. [Available from: https://www.nice.org.uk/process/pmg19/chapter/the-appraisal-process\#evidencereview.

34. Centre for Reviews and Dissemination. Systematic Reviews CRD's guidance for undertaking reviews in health care: CRD, University of York; 2009 [Available from: https://www.york.ac.uk/media/crd/Systematic_Reviews.pdf.

35. Joanna Briggs Institute. Joanna Briggs Institute Reviewer's Manual Adelaide: University of Adelaide; 2017 [Available from: https://reviewersmanual.joannabriggs.org/

36. Cobiac LJ, Vos T, Barendregt JJ. Cost-Effectiveness of Interventions to Promote Physical Activity: A Modelling Study. Plos Medicine 2009;6(7) doi: ARTN e1000110 10.1371/journal.pmed.1000110

37. Zapata-Diomedi B, Knibbs LD, Ware RS, et al. A shift from motorised travel to active transport: What are the potential health gains for an Australian city? PLoS ONE 2017;12(10):e0184799. doi: https://dx.doi.org/10.1371/journal.pone.0184799

38. Nshimyumukiza L, Durand A, Gagnon M, et al. An economic evaluation: Simulation of the cost-effectiveness and cost-utility of universal prevention strategies against osteoporosis-related fractures. J Bone Miner Res 2013;28(2):383-94. doi: 10.1002/jbmr.1758 [published Online First: 2012/09/20]

39. Goyder E, Hind D, Breckon J, et al. A randomised controlled trial and cost-effectiveness evaluation of 'booster' interventions to sustain increases in physical activity in middle- 
aged adults in deprived urban neighbourhoods. Health Technology Assessment 2014;18(13):1-+. doi: 10.3310/hta18130

40. Guo JY, Gandavarapu S. An economic evaluation of health-promotive built environment changes. Prev Med 2010;50 Suppl 1:S44-9. doi: 10.1016/j.ypmed.2009.08.019 [published Online First: 2009/10/21]

41. Cradock AL, Barrett JL, Kenney EL, et al. Using cost-effectiveness analysis to prioritize policy and programmatic approaches to physical activity promotion and obesity prevention in childhood. Prev Med 2017;95:S17-S27. doi:

10.1016/j.ypmed.2016.10.017

42. Gc VS, Suhrcke M, Hardeman W, et al. Cost-Effectiveness and Value of Information Analysis of Brief Interventions to Promote Physical Activity in Primary Care. Value Health 2018;21(1):18-26. doi: 10.1016/j.jval.2017.07.005 [published Online First: 2018/01/07]

43. Gulliford M, Charlton J, Bhattarai N, et al. Social and material deprivation and the costeffectiveness of an intervention to promote physical activity: cohort study and Markov model. Journal of Public Health 2014;36(4):674-83. doi: 10.1093/pubmed/fdt132

44. Munro JF, Nicholl JP, Brazier JE, et al. Cost effectiveness of a community based exercise programme in over 65 year olds: cluster randomised trial. J Epidemiol Commun $\mathrm{H}$ 2004;58(12):1004-10. doi: 10.1136/jech.2003.014225

45. Roux L, Pratt M, Lee IM, et al. Does age modify the cost-effectiveness of communitybased physical activity interventions? J Phys Act Health 2015;12(2):224-31. doi: 10.1123/jpah.2013-0167 [published Online First: 2014/05/20]

46. Roux L, Pratt M, Tengs TO, et al. Cost effectiveness of community-based physical activity interventions. Am J Prev Med 2008;35(6):578-88. doi: 10.1016/j.amepre.2008.06.040 [published Online First: 2008/11/13]

47. Anokye NK, Lord J, Fox-Rushby J. Is brief advice in primary care a cost-effective way to promote physical activity? Brit J Sport Med 2014;48(3):202-06. doi: 10.1136/bjsports-2013-092897

48. Barrett JL, Gortmaker SL, Long MW, et al. Cost Effectiveness of an Elementary School Active Physical Education Policy. American Journal of Preventive Medicine 2015;49(1):148-59. doi: 10.1016/j.amepre.2015.02.005

49. Cavill N, Muller, L., Mulhall, C. \& Rutter, H. Cycling demonstration towns: A costeffective investment to promote physical activity. Obesity Reviews 2011;12 
50. Dallat MAT, Soerjomataram I, Hunter RF, et al. Urban greenways have the potential to increase physical activity levels cost-effectively. Eur J Public Health 2014;24(2):19095. doi: 10.1093/eurpub/ckt035

51. Frew EJ, Bhatti M, Win K, et al. Cost-effectiveness of a community-based physical activity programme for adults (Be Active) in the UK: an economic analysis within a natural experiment. Br J Sports Med 2014;48(3):207-12. doi: 10.1136/bjsports-2012091202 [published Online First: 2012/07/17]

52. Over EA, Wendel-Vos GW, van den Berg M, et al. Cost-effectiveness of counseling and pedometer use to increase physical activity in the Netherlands: a modeling study. Cost Eff Resour Alloc 2012;10(1):13. doi: 10.1186/1478-7547-10-13 [published Online First: 2012/09/26]

53. Pringle A, Cooke C, Gilson N, et al. Cost-effectiveness of interventions to improve moderate physical activity: A study in nine UK sites. Health Education Journal 2010;69(2):211-24. doi: 10.1177/0017896910366790

54. Stahl JE 2008. Modelling methods for pharmacoeconomics and health technology assessment: an overview and guide. Pharmacoeconomics 26:131-148

55. Sculpher M, Fenwick E, Claxton K 2000. Assessing quality in decision analytic cost-effectiveness models. A suggested framework and example of application. Pharmacoeconomics 17:461-477

56. Vanni T, Karnon J, Madan J, et al. Calibrating models in economic evaluation: a sevenstep approach. Pharmacoeconomics 2011;29(1):35-49. doi: 10.2165/11584600000000000-00000 [published Online First: 2010/12/15]

57. Lacey B, Golledge J, Yeap BB, et al. Physical activity and vascular disease in a prospective cohort study of older men: The Health In Men Study (HIMS). BMC geriatr 2015;15:164. doi: 10.1186/s12877-015-0157-2 [published Online First: 2015/12/15]

58. Sculpher MJ, Claxton K, Drummond M, et al. Whither trial-based economic evaluation for health care decision making? Health Economics 2006;15(7):677-87. doi: 10.1002/hec. 1093

59. Cookson R, Drummond, M., \& Weatherly, H. Explicit incorporation of equity considerations into economic evaluation of public health interventions. Health Economics, Policy and Law 2009;4(2):231-45. doi: https://doi.org/10.1017/S1744133109004903 
60. National Institute for Health and Care Excellence. Guide to the methods of technology appraisal 20132013 [Available from:

https://www.nice.org.uk/process/pmg9/chapter/the-reference-case\#exploringuncertainty.

61. Evers S, Goossens M, de Vet H, van Tulder M, Ament A. Criteria list for assessment of methodological quality of economic evaluations: Consensus on Health Economic Criteria. Int J Technol Assess Health Care. 2005;21(2):240-245.

62. Ghislaine A.P.G. van Mastrigt, Mickaël Hiligsmann, Jacobus J.C. Arts, Pieter H. Broos, Jos Kleijnen, Silvia M.A.A. Evers \& Marian H.J.M. Majoie (2016) How to prepare asystematic review of economic evaluations for informing evidence-based healthcare decisions: a five-step approach (part 1/3), Expert Review of Pharmacoeconomics \& Outcomes Research, 16:6, 689-704, DOI: 10.1080/14737167.2016.1246960

63. Philips Z, Bojke L, Sculpher M, Claxton K, Golder S. Good practice guidelines for decision-analytic modelling in health technology assessment: a review and consolidation of quality assessment. Pharmacoeconomics. 2006;24(4):355-371. doi:10.2165/00019053-200624040-00006

64. Husereau D, Drummond M, Petrou S, et al. Consolidated health economic evaluation reporting standards (CHEERS) — explanation and elaboration: a report of the ISPOR Health Economic Evaluations Publication Guidelines Good Reporting Practices Task Force. Value Health. 2013;16(2):231-250.

65. Haji Ali Afzali H, Bojke L, Karnon J. Improving Decision-Making Processes in Health: Is It Time for (Disease-Specific) Reference Models? Appl Health Econ Health Policy 2019 doi: 10.1007/s40258-019-00510-6 [published Online First: 2019/08/23]

66. Amarasinghe AK. Cost-effectiveness implications of GP intervention to promote physical activity: evidence from Perth, Australia. Cost Eff Resour Alloc 2010;8:10. doi: 10.1186/1478-7547-8-10 [published Online First: 2010/05/14]

67. Beale SJ, Bending MW, Trueman P, et al. Should we invest in environmental interventions to encourage physical activity in England? An economic appraisal. Eur J Public Health 2012;22(6):869-73. doi: 10.1093/eurpub/ckr151

68. De Smedt D, De Cocker K, Annemans L, et al. A cost-effectiveness study of the community-based intervention '10 000 Steps Ghent'. Public Health Nutrition 2012;15(3):442-51. doi: 10.1017/S1368980011001716

69. Montes F, Sarmiento OL, Zarama R, et al. Do Health Benefits Outweigh the Costs of Mass Recreational Programs? An Economic Analysis of Four Ciclovia Programs. 
Journal of Urban Health-Bulletin of the New York Academy of Medicine 2012;89(1):153-70. doi: 10.1007/s11524-011-9628-8

70. Moodie M, Haby M, Galvin L, et al. Cost-effectiveness of active transport for primary school children - Walking School Bus program. Int J Behav Nutr Phy 2009;6 doi: Artn 63 10.1186/1479-5868-6-63

71. Verhoef TI, Trend V, Kelly B, et al. Cost-effectiveness analysis of offering free leisure centre memberships to physically inactive members of the public receiving state benefits: a case study. BMC Public Health 2016;16:616. doi: 10.1186/s12889-0163300-x [published Online First: 2016/07/28]

72. Wang G, Macera, C. A., Scudder-Soucie, B., Schimd, T., Pratt, M. \& Buchner, D. A costbenefit analysis of physical activity using bike/pedestrian trails. Health Promotion Practice 2005(6):174-9. 